\title{
Stellar Populations in the Outer Disk and Halo of the Spiral Galaxy M101
}

\author{
J. Christopher Mihos ${ }^{1}$ (D), Patrick R. Durrell ${ }^{2}$ (D) , John J. Feldmeier ${ }^{2}$ (D), Paul Harding ${ }^{1}$ (D), and Aaron E. Watkins ${ }^{1,3}$ \\ ${ }^{1}$ Department of Astronomy, Case Western Reserve University, Cleveland, OH 44106, USA \\ ${ }^{2}$ Department of Physics and Astronomy, Youngstown State University, Youngstown, OH 44555, USA \\ ${ }^{3}$ University of Oulu, Astronomy Research Unit, FI-90014 Oulu, Finland \\ Received 2018 April 13; revised 2018 June 13; accepted 2018 June 15; published 2018 July 26
}

\begin{abstract}
We use deep Hubble Space Telescope imaging in the outskirts of the nearby spiral M101 to study stellar populations in the galaxy's outer disk and halo. Our Advanced Camera for Surveys (ACS) field lies 17!.6 (36 kpc) from the center of M101 and targets the blue "NE Plume" of M101's outer disk, while the parallel Wide Field Camera 3 (WFC3) field lies at a distance of 23!3 (47 kpc) to sample the galaxy's stellar halo. The WFC3 halo field shows a well-defined red giant branch characterized by low metallicity $([M / H]=-1.7 \pm 0.2)$, with no evidence of young stellar populations. In contrast, the ACS disk field shows multiple stellar populations, including a young main sequence, blue and red helium-burning stars, and old RGB and asymptotic giant branch (AGB) populations. The mean metallicity of these disk stars is quite low: $[M / H]=-1.3 \pm 0.2$ for the RGB population, and $-1.15 \pm 0.2$ for the younger helium-burning sequences. Of particular interest is a bunching of stars along the BHeB sequence, indicative of an evolving cohort of massive young stars. We show that the young stellar populations in this field are well-described by a decaying burst of star formation that peaked $\sim 300-400$ Myr ago, along with a more extended star formation history to produce the older RGB and AGB populations. These results confirm and extend the results from our previous deep surface photometry of M101's outer disk, providing an important cross-check on stellar population studies using resolved stellar populations versus integrated light photometry. We discuss our results in the context of halo formation models and the interaction history of M101 and its companions.
\end{abstract}

Key words: galaxies: halos - galaxies: individual (M101) - galaxies: interactions - galaxies: spiral - galaxies: stellar content

\section{Introduction}

The diffuse outskirts of disk galaxies are shaped by a wide variety of physical processes linked to the evolution of galaxies. Ongoing, low-level star formation-as suggested by the extended ultraviolet (XUV) disks seen in many spiral galaxies (e.g., Gil de Paz et al. 2005; Thilker et al. 2007; Lemonias et al. 2011)—can gradually build the stellar populations over time. Interactions and accretion can deposit stars into the outer disk and halo (e.g., Bullock \& Johnston 2005; Martínez-Delgado et al. 2008, 2009; Villalobos \& Helmi 2008; Cooper et al. 2010; Ruiz-Lara et al. 2016), or trigger new star formation in the disk outskirts (e.g., Werk et al. 2010; Mihos et al. 2013; Bush et al. 2014). Tidal heating by galaxy interactions can also drive metal-rich stars outward from the inner disk (Walker et al. 1996; Younger et al. 2007); secular processes, such as radial migration, can scatter inner disk stars outward as well (Sellwood \& Binney 2002; Debattista et al. 2006; Roškar et al. 2008a, 2008b). These processes imprint signatures on the spatial distribution, kinematics, ages, and metallicities of the outer disk stellar populations that can be used to reconstruct the evolutionary history of the outer disk.

However, studying the stellar component of galaxy outskirts has proved particularly challenging. While the distribution and kinematics of the extended HI disk can be probed relatively simply via $21 \mathrm{~cm}$ line emission mapping, the surface brightness of the outer stellar component $\left(\mu_{V} \gtrsim 26\right.$ mag $\left.\operatorname{arcsec}^{-2}\right)$ is well below that of the night sky, requiring very deep and accurate surface photometry to measure its structure and optical colors (e.g., Bakos et al. 2008; Erwin et al. 2008; Watkins et al. 2014, 2016; Zheng et al. 2015; Merritt et al. 2016; Peters et al. 2017). A complementary approach is available for nearby galaxies
( $\lesssim 10 \mathrm{Mpc})$ : the study of the discrete stellar populations via deep Hubble Space Telescope (HST) imaging of individual stars in a galaxy's disk. The distribution of stars along the color-magnitude diagram (CMD) is sensitive to the age and metallicity of the underlying stellar population, and can be used to trace the star-forming history and metallicity evolution of the galaxy (e.g., Williams et al. 2009; 2011; Rejkuba et al. 2005; Dalcanton et al. 2009; McQuinn et al. 2010, 2011, 2012; Radburn-Smith et al. 2011; Bernard et al. 2015; Bruzzese et al. 2015; Monachesi et al. 2016).

As powerful as the discrete stellar population technique is, its ability to probe distant galaxies is limited by by the need to actually resolve individual stars. Ground-based studies have been restricted to relatively nearby galaxies within the Local Volume (e.g., Brooks et al. 2004; Davidge 2006, 2010; Harris \& Zaritsky 2009; Ibata et al. 2014); HST studies have pushed as far out as the Virgo Cluster (e.g., Ferguson et al. 1998; Harris et al. 1998; Caldwell 2006; Durrell et al. 2007; Williams et al. 2007; Bird et al. 2010), but typically only detect the brightest RGB stars. Studying the fainter populations becomes prohibitively expensive in terms of telescope time. At even larger distances, studies of stellar populations in galaxy outskirts are limited to deep surface photometry, which provides much weaker information due to the poor discrimination broadband colors have to ages greater than a few Gyr, coupled with the well-known age-metallicity degeneracy. While star formation rates can be readily probed through deep $\mathrm{H} \alpha$ or FUV imaging (see, e.g., the review by Kennicutt \& Evans 2012), these are sensitive only to timescales of $\lesssim 100 \mathrm{Myr}$, the lifetime of the massive UV-producing stars. Obtaining the multicolor imaging or spectrophotometry that 
yields more nuanced information over a wider range of stellar ages is largely prohibitive at such low surface brightness. The limited reach of discrete stellar population work, coupled with the degeneracies of integrated light techniques, leaves us with a dissatisfying seam in our studies of the star-forming histories of galaxies. Much of our detailed information regarding the Local Group comes from discrete stellar populations, while our more general understanding of the star-forming histories of galaxies comes from studying large samples of more distant galaxies via imaging and spectrophotometry of their integrated light. The differing systematics of these two techniques means that connecting lessons learned from the Local Group to those from more distant galaxies remains uncertain.

The nearby spiral galaxy M101 (NGC 5457, $D=6.9 \mathrm{Mpc}$; see Matheson et al. (2012) and references therein) provides a particularly important test case for studying the evolution of disk outskirts and connecting deep surface photometry studies with discrete stellar population work. M101 is the largest member of a small galaxy group (Geller \& Huchra 1983; Tully 1988), an environment where interactions with companions can drive significant evolution in the galaxy's disk. Deep far-UV imaging from GALEX has shown M101 to be an XUV disk (Thilker et al. 2007), with star formation occurring at a large radius well outside the bright inner disk. M101 has been well-studied at many wavelengths, giving us a comprehensive view of the galaxy's structure, kinematics, and star-forming properties. Its proximity also makes it an optimal target for studying the stellar populations of its outer disk and halo via discrete stellar population techniques.

The complicated outer structure of M101's disk was first revealed in deep, wide-field surface photometry of the galaxy by Mihos et al. (2013; hereafter M+13). Beyond 12!.5 (25 kpc), the outer disk shows two distinct two stellar plumes-dubbed the Northeast (NE) Plume and Eastern Spur by $\mathrm{M}+13$ - suggestive of tidal features from a past encounter. The NE Plume, in particular, showed extremely blue colors $(B-V \approx 0.2)$, but little ongoing star formation-as evidenced by the lack of farUV light. This led $\mathrm{M}+13$ to propose that the outer disk had experienced a weak burst of star formation that peaked 250-350 Myr ago and had since largely died out. However, due to the age-metallicity degeneracy of broadband imaging, little could be said regarding the metallicity of the population, the robustness of the inferred star-forming history, or the presence of any underlying older populations. Aside from the stellar plumes, little additional light was detected beyond the galaxy's outer disk, a fact that led van Dokkum et al. (2014) to propose that M101 had a very anemic stellar halo, nearly an order of magnitude lower in stellar mass fraction than that of the Milky Way or M31.

Discrete imaging of M101's outskirts using HST has the potential to address many of the issues surrounding the evolutionary history of M101's disk and the presence of halo stellar populations. The colors and number counts of stars of differing evolutionary phases along the CMD_along the main sequence, in helium-burning phases, or on the red giant branch or asymptotic giant branch (AGB) - will give a much more robust measure of the star formation history of the outer disk and provide, for the first time, metallicity estimates of the galaxy's stellar populations. Furthermore, because star count techniques have the ability to go to lower equivalent surface brightness than deep surface photometry (e.g., Ibata et al. 2014; Okamoto et al. 2015; Crnojević et al. 2016), we can also make a direct measure of the density of M101's stellar halo. Finally, aside from learning about the evolutionary history of M101 itself, follow-up HST imaging of discrete populations in its outer disk could permit valuable cross-comparisons of the deep surface photometry and discrete stellar population imaging techniques.

In this paper, we follow up on the imaging of $\mathrm{M}+13$ by studying the NE Plume, using HST Advanced Camera for Surveys (ACS) imaging that extends deep enough to detect not just the RGB stars and massive helium-burning stars, but the young main sequence population as well. By constructing a deep CMD of the stellar populations, we can use isochrone matching and CMD modeling techniques to infer the metallicity distribution of stars in M101's outer disk and probe the star-forming history of the stellar plumes with much finer discriminatory power than was possible using the broadband imaging techniques of $\mathrm{M}+13$. We also use Wide Field Camera 3 (WFC3) imaging of a blank field near the NE Plume to search for and characterize any stellar populations associated with M101's stellar halo, in order to assess the conjecture that M101 has an anomalously weak halo component. Finally, we also make a direct comparison of these results to deep ground-based surface photometry of M101, in order to connect these two complementary methods for studying stellar populations in galaxies.

\section{Observations and Data Reduction}

\subsection{Field Selection and Observations}

To sample the stellar populations in M101's outer disk and halo, we obtained observations of a pair of fields (see Figure 1) via both the Wide Field Channel (WFC) of the ACS and the UVIS channel of the WFC3 on-board the HST, as program GO-13701. The primary ACS field (covering $\approx 3 ! 4 \times 3 ! 4$ ) lies on the NE Plume, centered at $\alpha=14^{\mathrm{h}} 04^{\mathrm{m}} 50$ s. $62, \delta=+54^{\circ} 31^{\prime} 19^{\prime \prime} .1$ (J2000.0), with a projected distance of $17 ! 6(36 \mathrm{kpc})$ from the center of the galaxy. The field was chosen to avoid the most actively star-forming regions of the NE Plume, and accordingly is somewhat redder $(B-V=0.31)$ than the Plume as a whole $(B-V=0.21 ; \mathrm{M}+13)$. The parallel WFC3 field, located at $\alpha=14^{\mathrm{h}} 05^{\mathrm{m}} 31^{\mathrm{s}} .93, \delta=+54^{\circ} 32^{\prime} 26^{\prime \prime} .2(\mathrm{~J} 2000.0)$, covers a region outside the disk where no diffuse starlight was detected by $\mathrm{M}+13$ down to a surface brightness limit of $\mu_{B}=29.5 \mathrm{mag} \mathrm{arcsec}^{-2}$. The field lies at a projected distance of $23.3(47 \mathrm{kpc})$ from the center of M101; it will serve as a control/background field for the ACS imaging, in addition to providing a glimpse into the stellar populations of M101's halo.

The two fields were observed over 26 orbits during 2015 September 21-26, with two images per orbit. The depth of our imaging is driven by the desire to detect not only the luminous old RGB and young helium-burning stars, but also the young main sequence population down to an age of $\sim 400 \mathrm{Myr}$. At M101's distance, this age corresponds to a main sequence turnoff at $\mathrm{F} 814 \mathrm{~W} \approx 28.5$. The primary ACS observations were split into two orbit visits with an exposure series of (1320, 1320, $1415,1415 \mathrm{~s}$ ) per visit. The F606W observations included seven visits, for a total exposure time of $38,290 \mathrm{~s}$; the F814W observations were taken over six visits, for a total exposure time of $32,820 \mathrm{~s}$. The exposure time series for the parallel WFC3 observations were $(1400,1400,1450,1450 \mathrm{~s})$, with total exposure times of $34,200 \mathrm{~s}$ for the F606W observations over six visits, and $39,900 \mathrm{~s}$ for the F814W observations over seven 

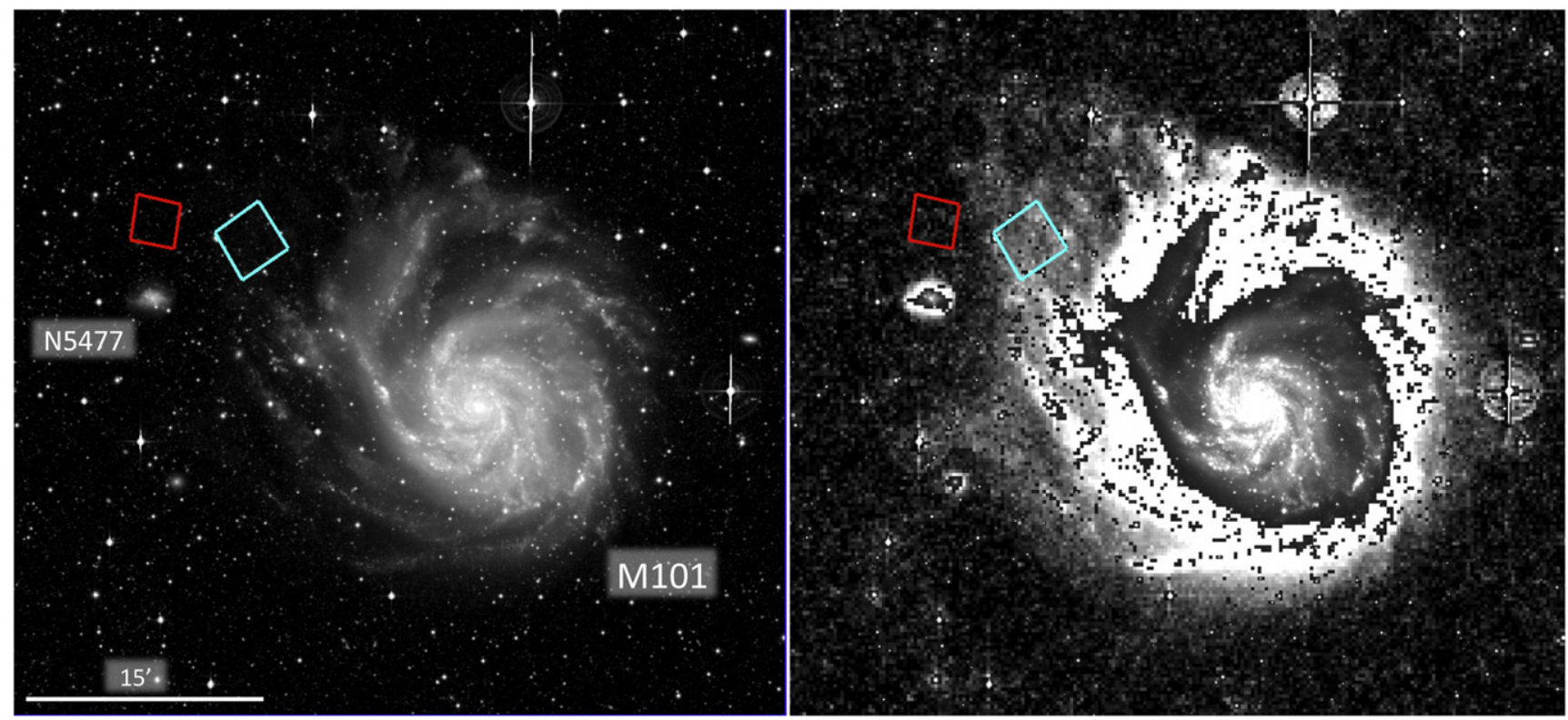

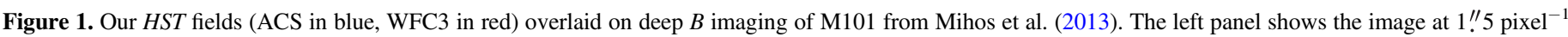

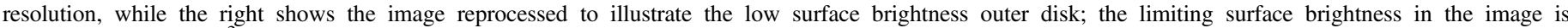
$\mu_{B}=29.5 \mathrm{mag} \mathrm{arcsec}^{-2}$ (see Mihos et al. 2013 for details).

visits - the longer exposures times for F814W were needed to account for the lower quantum efficiency of the WFC3 camera in F814W. In each visit, we used a customized four-point sub-pixel DITHER-BOX pattern to maximize the sampling of the PSF over both cameras. Each visit was dithered with three larger $\Delta x=20$ pixel $\left(\sim 1^{\prime \prime}\right)$ offsets to effectively remove bad pixels, with this pattern repeated at an additional $\Delta y=60$ pixel $\left(\sim 3^{\prime \prime}\right)$ shift to cover the ACS chip gap, allowing for a contiguous study of the stellar populations in our fields. We retrieved the calibrated, CTE-corrected . $f l c$ images for both cameras in 2016 May, in order to make use of images with the best possible calibration files applied.

\subsection{Point Source Photometry}

We used the ACS module of the DOLPHOT 2.0 software package (Dolphin 2000) for the photometric analysis of the ACS field; this package is specifically designed for pointsource photometry of objects in the individual CTE-corrected . $f l c$ images. Object detection and photometry were performed simultaneously on all exposures, using a deep image stack derived from multidrizzling (using drizzlepac 2.0) all 28 individual F814W images for use as the reference image. The DOLPHOT parameters were set to the recommended values for ACS/WFC images as given in the DOLPHOT User's Guide, ${ }^{4}$ which includes PSF-fitting using pre-derived PSFs, as well as the calculation and application of aperture corrections. Sky values were determined using large regions around each object, as the images are not crowded and have no large-scale background sky variations. The instrumental magnitudes were converted to the VEGAMAG HST photometric system by adopting updated F606W and F814W zeropoints for 2016 data products.

Photometric reductions on the individual WFC3 images were conducted with DOLPHOT in a method similar to the ACS reductions. Jang \& Lee (2015), however, note small differences

\footnotetext{
4 http://purcell.as.arizona.edu/dolphot/
}

$(\lesssim 0.05 \mathrm{mag}$ ) between the F606W and F814W VEGAMAG values between the two cameras. Thus, to facilitate a cleaner comparison between the two data sets, we have applied their conversions to put the WFC3 magnitudes on the ACS system. We refer to magnitudes in the ACS VEGAMAG system by their filter names (F606W and F814W); in cases when we need to convert to the Johnson-Cousins systems, we refer to those filters as $V$ and $I$.

We determined the photometric completeness limits and associated errors in our point-source photometry by using DOLPHOT to add and remeasure 100,000 artificial stars in each of the ACS and WFC3 image sets. We used input artificial stars with a wide range of magnitudes $(24.0<\mathrm{F} 606 \mathrm{~W}<31.0)$ and colors $(-1.0<\mathrm{F} 606 \mathrm{~W}-\mathrm{F} 814 \mathrm{~W}<2.5)$, in order to investigate completeness for all of the possible stellar populations in our CMDs. In processing the artificial star photometry, we also apply the same photometric cuts on detection threshold and image shape that we use on the real data (see next section), in order to ensure the best measure of photometric errors and completeness in our extracted CMDs. In the ACS imaging, we find a $90 \%(50 \%)$ completeness limit of 28.6 (29.4) in F606W and 27.8 (28.6) in F814W. For the WFC3 imaging, the $90 \%(50 \%)$ limits are $28.6(29.5)$ in F606W and 27.7 (28.6) in F814W. We also use the artificial star tests to determine photometric uncertainty, with characteristic error bars as a function of magnitude shown in Figure 2. At faint magnitudes, near the $50 \%$ completeness limits, we find a slight blueward shift of $\sim 0.06 \mathrm{mag}$ in the F606W - F814W colors, illustrated by the shift of the Figure 2 error bars off the $\mathrm{F} 606 \mathrm{~W}-\mathrm{F} 814 \mathrm{~W}=-1$ axis line. However, this shift drops to less than $0.01 \mathrm{mag}$ at brighter magnitudes $(\mathrm{F} 814 \mathrm{~W}<26.5)$, and is always much smaller than the photometric uncertainty at all magnitudes.

\subsection{Color-Magnitude Diagrams}

To create the F606W, F814W CMDs for each of our fields, we first applied a series of criteria to extract the best possible 


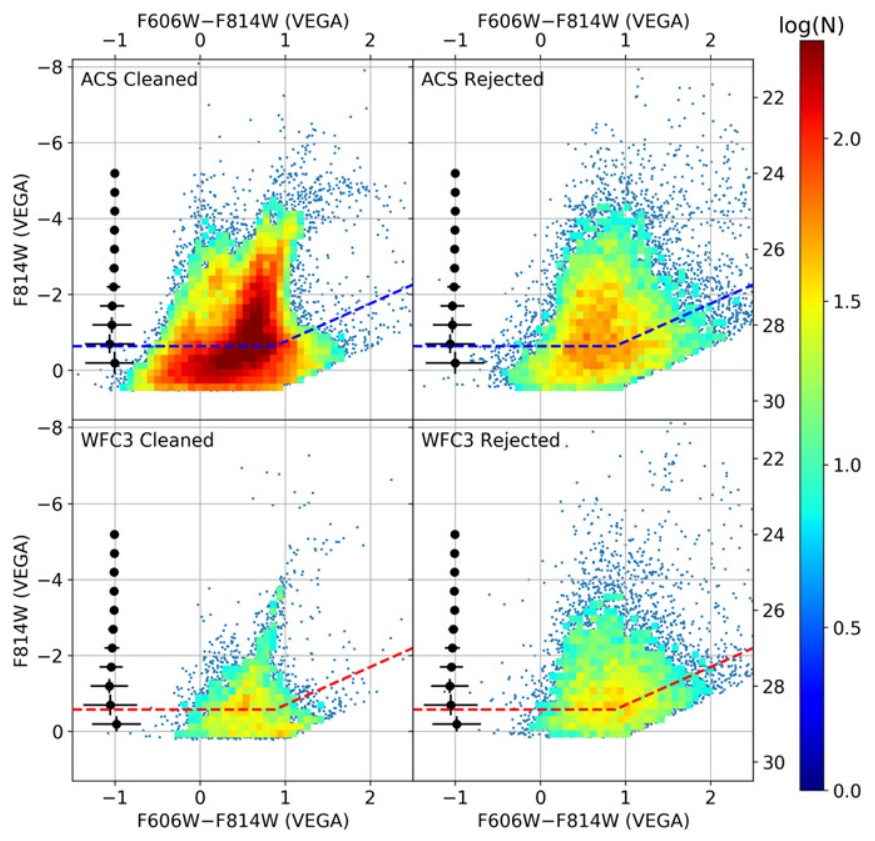

Figure 2. The photometrically cleaned stellar sources (left panels) and the rejected objects (right panels) plotted on the F606W, F814W color-magnitude diagram. The CMDs show no structure in the distribution of rejected sources that would indicate over-rejection of stellar sources in M101. Characteristic error bars are shown in each CMD, while the dashed lines show the 50\% completeness depth of the data. In these and all subsequent CMD plots, the right axis shows the observed apparent magnitude of the objects, while the left axis shows the absolute magnitude of the source at the M101 distance (6.9 Mpc).

photometry for those point sources detected in both the F606W and F814W images. Only those objects with DOLPHOT object TYPE $=1$ ("good star") and combined $\mathrm{S} / \mathrm{N}$ values $>3.0$ in both filters were retained for further analysis. While these cuts remove the lowest $\mathrm{S} / \mathrm{N}$ photometry and obvious non-stellar objects, we used additional criteria from the DOLPHOT output to remove as many non-stellar objects as possible while keeping those point sources with the best available photometry. To this end, we retained only those objects that were measured on at least eight of the images in both filters $\left(N_{\mathrm{F} 814 \mathrm{~W}}>8\right.$, $N_{\text {F606W }}>8$ ) and have CROWD $<0.4$ mag and goodness-of fit $\mathrm{CHI}<2.0$ in both filters. Finally, we used only those objects with sharpness values consistent with point sources $\mid$ sharp $\mid<0.08+0.25 e^{(\mathrm{F} 814 \mathrm{~W}-28.5)}$. This function was used to select only point sources, and is similar to that used to successfully identify M81 halo star populations in Durrell et al. (2010). The best-choice coefficients for the function were determined empirically through visual inspection of objects on the drizzled/stacked F606W and F814W images, down to $\mathrm{F} 814 \mathrm{~W}=27$. To ensure that we are not being overly aggressive and removing true point sources, we examine the effect of this criterion on our artificial star tests, where we find that less than $1 \%$ of the artificial stars brighter than our $50 \%$ completeness limits are being rejected by this choice.

The final, cleaned CMDs for each of the ACS and WFC3 fields (after application of all criteria above) are presented in Figure 2. One potential concern is that our rejection of nonstellar sources may be too aggressive and eliminate bona fide M101 stars in addition to the contaminating sources. To test this, Figure 2 also shows the CMDs for objects that did not pass the sharpness, CROWD, CHI, $N_{\mathrm{F} 606}$, and $N_{\mathrm{F} 814}$ criteria. If we are overly aggressive in our rejection of non-stellar sources, we should see stellar populations sequences (such as RGB or helium-burning stars) in the rejected-source CMDs. However, inspection of these rejected objects shows no obvious population sequences and very little structural similarity to the cleaned stellar-source CMDs, giving us confidence that we are not overaggressively rejecting M101 stars in our photometric cleaning.

After all cuts, the ACS CMD has a total of 33,967 sources in it, with $8924(17,417)$ sources brighter than the ACS $90 \%$ (50\%) completeness limit. The WFC3 CMD has a total of 3513 sources, with 590 (1786) sources brighter than the WFC3 90\% $(50 \%)$ completeness limit.

\section{Background Source Estimation}

Given the depth of our observations, there is potential for significant contamination from both foreground Milky Way stars and unresolved background galaxies. Following the work of others (e.g., Radburn-Smith et al. 2011; Dalcanton et al. 2012; Monachesi et al. 2016), we first estimated foreground Milky Way contamination. Using both the Besançon (Robin et al. 2003) and TRILEGAL (Girardi et al. 2005; Girardi 2016) models in their default configurations, we simulated one square degree of Milky Way sources around the central coordinate of our target locations, to ensure well-populated CMDs. We then rescaled the counts to the actual area of the ACS and WFC3 fields, and used the photometric transformation of Sirianni et al. (2005) to transform the models' $V$ and $I$ photometry into F606W and F814W magnitudes, We found that the Besançon model and the TRILEGAL model both produced $\approx 37(25)$ Galactic foreground stars within our ACS (WFC3) field, down the adopted $90 \%$ completeness limit of $\mathrm{F} 814 \mathrm{~W} \approx 27.7$, corresponding to 3.2 stars per $\operatorname{arcmin}^{2}$ over the entire magnitude and color range. The expected number of foreground Galactic stars increases slightly at our 50\% completeness limit of $\mathrm{F} 814 \mathrm{~W} \approx 28.6$ (40 and 27 for the ACS and WFC3 fields, respectively). Below these limits, the two Galactic models diverge substantially, with the Besançon model predicting $70 \%$ more stars at fainter magnitudes. The large offset in predicted counts between the two Milky Way models at faint magnitudes has been noted by others (e.g., Fantin et al. 2017; Majewski et al. 2017), and is due to differing model assumptions in both stellar evolution and in the structural parameters of the Milky Way. However, these results show that, except for brighter regions of our CMDs that contain a small number of objects, the effect of the foreground contamination from Milky Way stars is negligible. Instead, as has been found by many others (e.g., Ferguson et al. 2000; Ellis \& Bland-Hawthorn 2007; Windhorst et al. 2011), for deep HST imaging, the dominant source of contamination is unresolved background galaxies. Although this contamination can be calculated approximately (e.g., Ellis \& Bland-Hawthorn 2007), for an independent assessment of background contamination and to act as a background field for the WFC3 pointing, we measure this contamination empirically from archival HST imaging data as described below.

Because of the extreme depth of our imaging, most of the background fields used in other studies of the outskirts of nearby galaxies (e.g., Radburn-Smith et al. 2011; Monachesi et al. 2016) have insufficient depth for our purposes. For our study, we need data that (1) have been taken using the ACS camera, (2) are as deep as our observations in both F606W and 
F814W, and (3) are not known to have a large number of foreground sources. Ideally, given the known degradation of the ACS detectors with time (e.g., Anderson \& Bedin 2010; Massey et al. 2014; Bohlin 2016 and references therein), it would also be beneficial to also use background fields taken close in time to our observations. Only a handful of data sets satisfy these criteria, from which we chose the Hubble Frontier Field parallel fields (Lotz et al. 2017) from the Abell 2744 imaging (GO 13495; PI J. Lotz; GO 13386; PI S. Rodney, GO 13389; PI B. Siana) to estimate the background contamination in our study of M101.

We note several possible concerns with using the Abell 2744 parallel field for background estimation. First, being projected only $\approx 6$ !. 0 (2 Mpc) from the cluster core, the field may contain an excess of sources from the cluster itself. However, Lee \& Jang (2016) compared the luminosity functions of objects in the parallel field to those in both the main Abell 2744 cluster field and the HUDF/XDF field (Illingworth et al. 2013) and found only a small excess of resolved galaxies in the Abell 2744 parallel field. Second, due to enhanced magnification from weak gravitational lensing, the apparent fluxes of the background sources may be enhanced. Castellano et al. (2016) and Lotz et al. (2017) discuss the weak-lensing magnification for the Abell 2744 parallel field and report a median magnification factor of $\approx 1.2$, depending on the exact position and redshift of the background source. This magnification bias affects our contamination estimate by boosting otherwise undetected sources above our detection threshold (e.g., Schmidt et al. 2009), and it may lead to a slight overestimate of the contamination in the M101 field. Third, by basing our background estimate on a single deep field with a small field of view, our estimate is likely to suffer from the effects of significant cosmic variance (e.g., Somerville et al. 2004; Trenti \& Stiavelli 2008).

With these caveats in mind, we extracted a subset of the total Abell 2744 parallel observations to best mimic the depth of our own science observations: we used all long F606W visits (eight in total, with five visits taken from the Frontier Fields, and three visits taken by other observers), and the first seven visits in F814W from the Frontier Fields. The F814W exposures consisted of two orbit visits with an exposure series of $(1125,1207,1307,1207 \mathrm{~s})$ or $(1225,1307,1507,1207 \mathrm{~s})$, with the corresponding F606W exposure series being identical in length. Both Frontier Fields ACS exposure series were effectively dithered by the primary WFC3/IR pattern "IR-DITHER-BLOB," and there was a small random offset between each visit (see Lotz et al. 2017 for full details). The additional F606W visits were observed at nearly the same roll angle as the Frontier Fields and consisted of four $1250 \mathrm{~s}$ exposures using the "WFC3-UVIS-DITHER-BOX" pattern. The total exposure times for our background field are 35,522 s in F814W and 40,430 s in F606W. Additional F814W images are available to increase the photometric depth in F814W, but we did not include since our goal is to mimic, as closely as possible, a background field that has photometric depth and signal-to-noise similar to our own M101 data. Given that we are limited by the availability of F606W background images, and that color information is critical for our analysis, it is unlikely that that the additional F814W exposures would substantially improve the background determination.

To extract the background estimate, we used procedures for image alignment, drizzling, object detection, and photometry

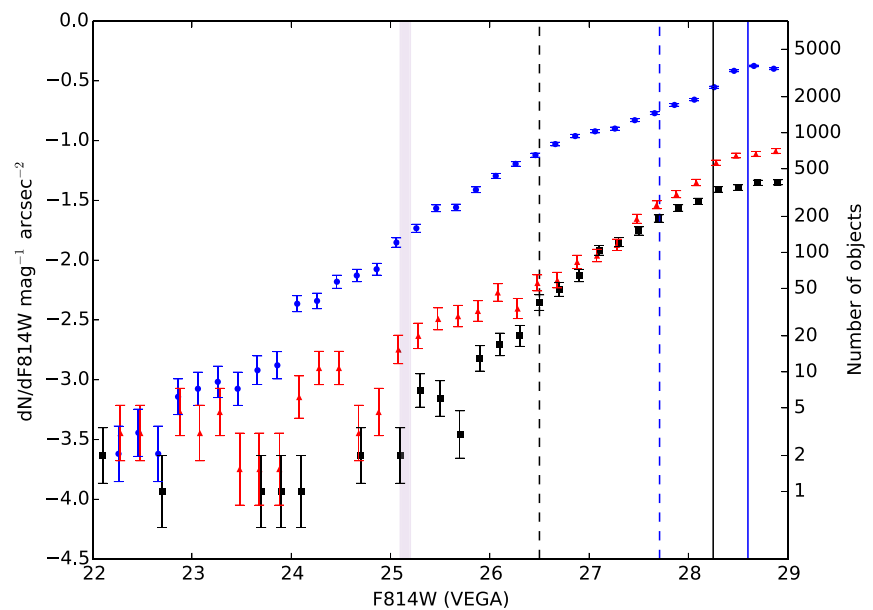

Figure 3. The observed F814W luminosity function of point sources in the M101 ACS field (blue circles), WFC3 field (red triangles), and background Abell 2744 parallel field (black squares), binned into 0.2 mag intervals. The shaded purple region shows the approximate location of the TRGB for M101. The dashed (solid) black and blue lines correspond to the $90 \%(50 \%)$ completeness limits for the background and ACS fields, respectively; the WFC3 completeness limits are within 0.06 mag of the ACS fields. For ease of comparison, small offsets of -0.02 mag and -0.04 mag have been applied to the WFC3 and ACS number counts bin centers, respectively.

identical to those used for the M101 ACS and WFC3 fields discussed in Section 2.2. We again used DOLPHOT for object detection and photometry on all images simultaneously, based on a deep image stack derived from multidrizzling all 28 individual $\mathrm{F} 814 \mathrm{~W}$ images together. The final area for the stacked background image was slightly larger than our M101 ACS field, at $\approx 11.95 \operatorname{arcmin}^{2}$. We also determined the photometric errors and completeness limits for the background field by adding and remeasuring 50,000 artificial stars in the same manner as for the M101 target fields, deriving 90\% (50\%) completeness limit of 27.8 (29.1) in F606W and 26.5 (28.3) in F814W for the background field. We then applied an identical selection criteria to the DOLPHOT output for the background field discussed in Section 2.3 (object type, $\mathrm{S} / \mathrm{N}$ values, number of frames detected, CROWD, CHI, and sharpness) to obtain the final CMD. We detected a total of 3613 point sources that met our selection criteria, with 197 (1383) sources brighter than the 90\% (50\%) F814W completeness limit.

Figure 3 shows the F814W luminosity function of all stellar sources in the background field that passed our selection criteria, comparing it to the corresponding F814W luminosity functions from the ACS and WFC3 fields targeting M101. There is a clear excess of sources in both the ACS and WFC3 M101 fields, compared to the background field. We also note a dip in the luminosity function in both fields just brightward of $\mathrm{F} 814 \mathrm{~W} \approx 25.2$, the approximate location of the tip of the red giant branch (TRGB) as seen in the CMDs of Figure 2. The presence of contaminants and AGB stars at brighter magnitudes makes this "TRGB edge" weaker than might be expected for a pure, extremely old stellar population. However, one magnitude brighter than the TRGB, we find only 338 (26) sources after background subtraction in the ACS (WFC3) field-while one magnitude fainter than the TRGB, there are 1311 (71) objects. Therefore, even without using any color information, we have a detection of the TRGB in both of our observed M101 fields. However, as the luminosity function extends to fainter magnitudes, the number of background sources and the systematic uncertainties discussed above increase significantly, 


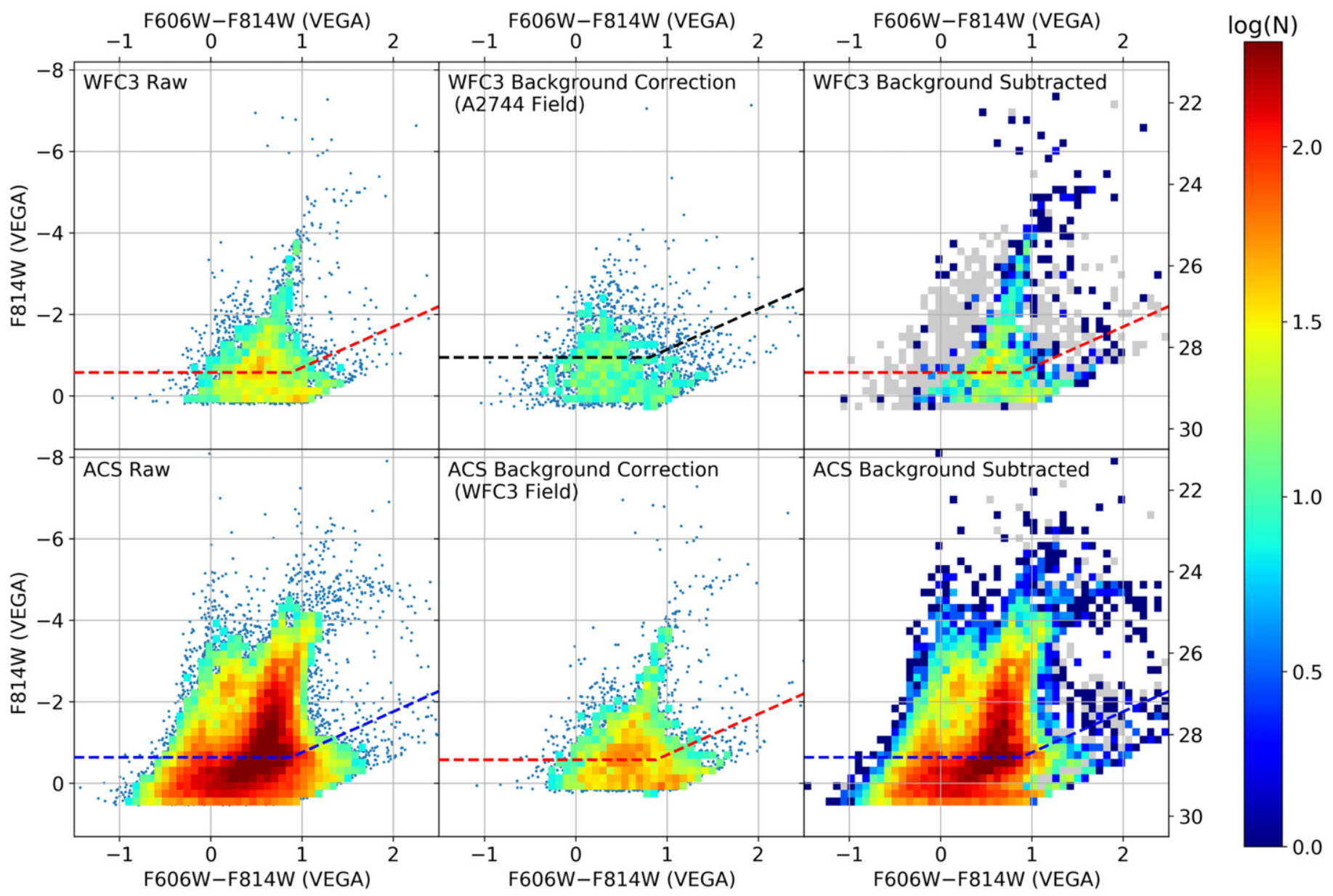

Figure 4. Extracted CMDs for the WFC3 (top) and ACS (bottom) fields. Left panels show the full CMDs; middle panels show the expected background contamination estimated using data from the Abell 2744 Flanking Field (for the WFC3 field) or the WFC3 field (for the ACS field); right panels show the background-subtracted CMDs. The color bar shows the number of stars in the binned regions of the CMDs; this color scale is used in all subsequent CMDs shown in this work. The 50\% completeness limits for each field are shown as dotted lines.

and the color distribution of the sources must be taken into account.

\section{Final CMDs and Stellar Populations}

Figure 4 shows the cleaned CMDs for the WFC3 and ACS fields, both before and after background subtraction, and after applying foreground extinction corrections of $A_{\mathrm{F} 606 \mathrm{~W}}=$ $0.024, A_{\mathrm{F} 814 \mathrm{~W}}=0.015$ from Schlafly \& Finkbeiner (2011). For the WFC3 field, the background correction is taken to be the A2744 CMD, downsampled in size to account for the different areas of the two fields. For the ACS field, we have two choices for a background: the A2744 field or the WFC3 field. In the ACS field, potential contamination comes from two major sources: unresolved background galaxies and stars in M101's stellar halo. Brighter than $\mathrm{F} 814 \mathrm{~W}=27$, these M101 halo stars dominate over background galaxies and have the potential to introduce a false RGB signal into the ACS CMD. Thus, for the ACS field, we adopt our parallel WFC3 CMD as the background estimate, with the important caveat that M101 halo stars could be even more prevalent in the ACS CMD, due to its smaller galactocentric radius; we examine this possibility in greater detail in Section 4.1. Because the WFC3 field has a smaller area than the ACS field, we upsample the WFC3 sources by randomly duplicating sources and adding a random scatter to their F606W and F814W magnitudes, taken from a uniform deviate spanning \pm 0.05 mag. For each of the ACS and WFC3 fields, after construction of the appropriate background estimate, the CMD and its background are both binned into color-magnitude bins of size $0.08 \times 0.2 \mathrm{mag}$, after which the background is subtracted off.

In the WFC3 field, the obvious RGB sequence visible in the raw CMD remains intact after subtraction, along with some hint of an intermediate-age AGB population extending to brighter and redder colors. Aside from that, we see no clear signature of any other stellar populations; the scattering of stars blueward of F606W $-\mathrm{F} 814 \mathrm{~W}=0.5$ and F814W $>26$ appears consistent with background contamination, as evident in the Abell 2744 flanking field. Indeed, blueward of the RGB, in a region spanning the color range $0.0<\mathrm{F} 606 \mathrm{~W}-\mathrm{F} 814 \mathrm{~W}<0.45$ and magnitude $-2.5<M_{\mathrm{F} 814 \mathrm{~W}}<-1.5$, counts in the Abell 2744 flanking field are actually a factor of two higher than the counts in the WFC3 M101 field (the effect of this can be seen in the backgroundsubtracted WFC3 CMD, where gray regions indicate oversubtraction). However, this region of the WFC3 is sparsely populated; along the RGB sequence, the oversubtraction is less significant, due to the increased counts on the RGB, but also because there are fewer sources in the background estimate on this portion of the CMD.

In the ACS field, after background subtraction, the CMD is largely unchanged from a qualitative perspective. The multiple stellar populations evident in the raw CMD remain and are marked in Figure 5. In addition to the RGB, we see a well- 


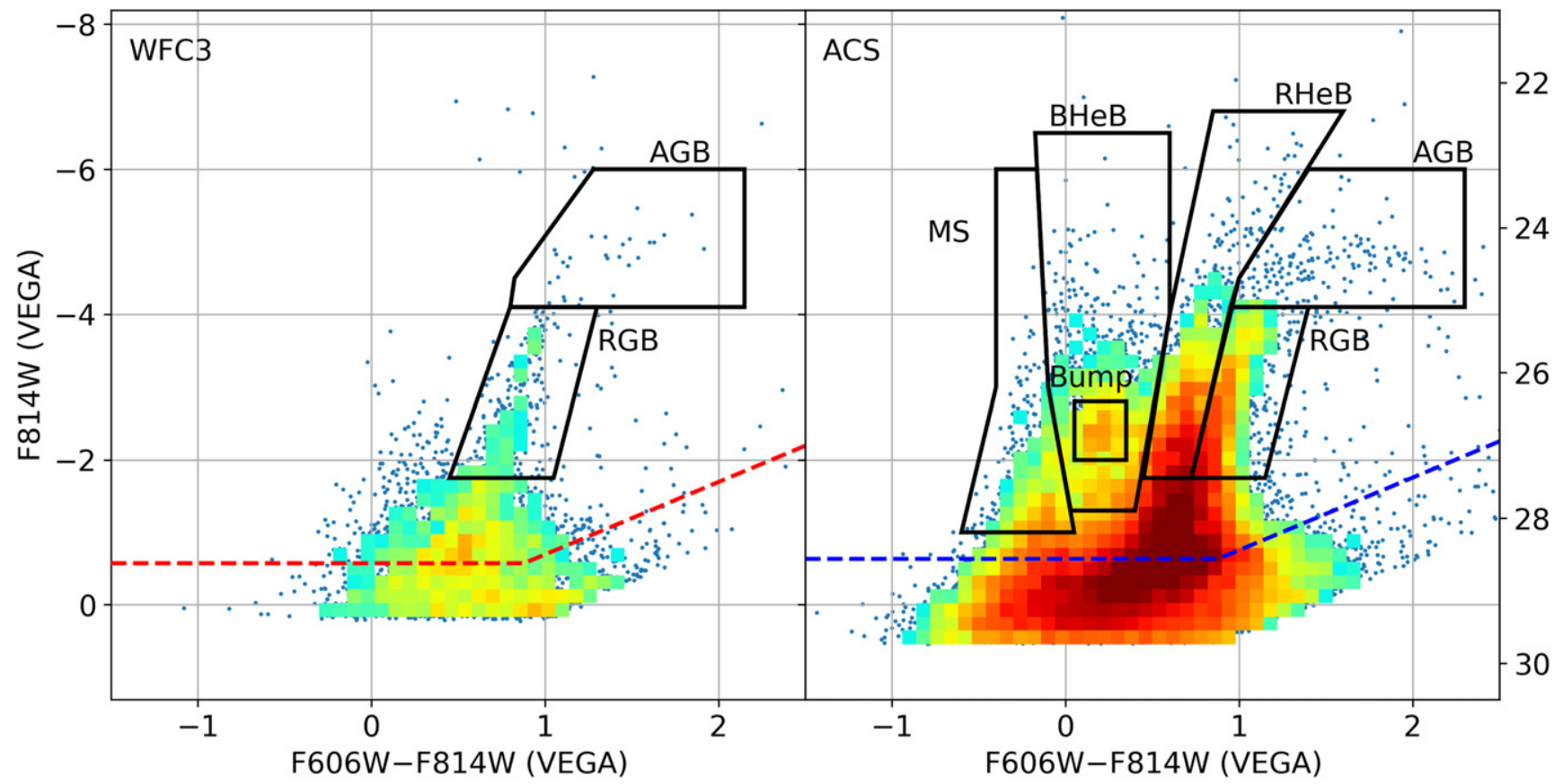

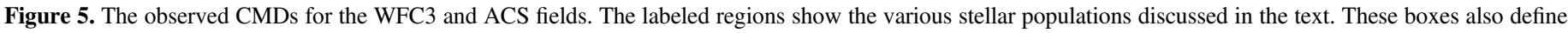
the photometric selection criteria used when performing the quantitative CMD analyses described in Section 4.

populated AGB sequence, red and blue helium-burning populations (RHeB and $\mathrm{BHeB}$ ), and the upper main sequence at $M_{\mathrm{F} 814 \mathrm{~W}} \sim-1.5$. The CMD appears qualitatively similar to those of metal-poor, star-forming dwarf galaxies (e.g., McQuinn et al. 2010), with one notable exception. In star-forming galaxies, the $\mathrm{BHeB}$ typically appears as a smooth sequence of stars extending between the RHeB and upper main sequence, but in the M101 ACS field, the BHeB sequence instead shows a strong concentration of stars at $\mathrm{F} 606 \mathrm{~W}-\mathrm{F} 814 \mathrm{~W}=0.2$ and $M_{\mathrm{F} 814 \mathrm{~W}}=-2.4$, suggestive of a cohort of stars evolving together with only a small spread in stellar age and metallicity.

In what follows, we use a combination of isochrone matching and CMD modeling to further explore the stellar populations in M101's halo and outer disk. Having demonstrated the effects of background correction in Figure 4, our subsequent analyses will focus on the uncorrected raw CMDs, so as to preserve as much information as possible in the data; in cases where background corrections are necessary for the analysis (such as for CMD modeling), we incorporate these corrections directly into the models, rather than working with the subtracted data sets.

\subsection{Old Stellar Populations in the ACS Disk and WFC3 Halo Fields}

We begin with a comparative analysis of the old stellar populations seen in the WFC3 and ACS fields. Both fields show a clear RGB sequence, with additional AGB stars evident above the RGB tip at $M_{\mathrm{F} 814 \mathrm{~W}} \sim-4$ (Figure 5). However, due to the paucity of AGB stars in each field, as well as uncertainties in stellar modeling of the AGB phase, we confine our quantitative analysis to the RGB sequences and defer a more general consideration of the AGB populations to Section 5. The RGB populations in the two fields are clearly distinct from one another in color, as can be seen in Figure 6, which shows the color distribution of stars within 0.5 mag of the TRGB. We show both a raw histogram of color and a
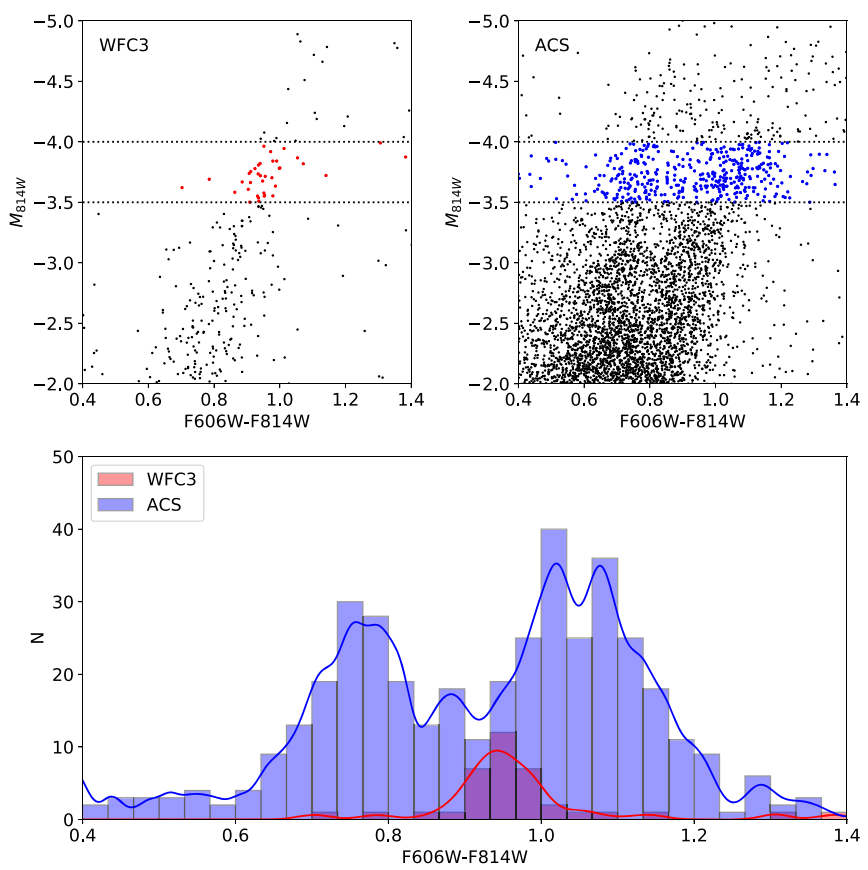

Figure 6. The F606W - F814W color distribution for stars within 0.5 mag of the tip of the RGB in the WFC3 and ACS fields. The selection is shown in the upper left (WFC3) and upper right (ACS) panels, while the bottom panel shows both a binned color histogram and a smoothed representation of the color distribution (see text for details).

smooth distribution function of color calculated as the sum of Gaussians with the means and dispersions set to be the measured color and color uncertainty of each star in the selection.

In the WFC3 field, the color distribution shows a single peak due to its old, metal-poor RGB, with median color $\mathrm{F} 606 \mathrm{~W}-\mathrm{F} 814 \mathrm{~W}=0.96 \pm 0.04$. In the ACS field, it shows two peaks; one at $\mathrm{F} 606 \mathrm{~W}-\mathrm{F} 814 \mathrm{~W} \sim 0.75$ due to the RHeB 
stars, and another at $\mathrm{F} 606 \mathrm{~W}-\mathrm{F} 814 \mathrm{~W} \sim 1.05$ due to the RGB. For RGB stars within the color range $0.95<$ F606W - F814W $<1.2$, the median RGB color is F606W $\mathrm{F} 814 \mathrm{~W}=1.07 \pm 0.07$, or about $0.1 \mathrm{mag}$ redder than that in the WFC3 field. ${ }^{5}$ The RGB population in the ACS field also shows a broader color spread, suggesting both a higher mean metallicity and a wider range of metallicities.

Indeed, the broad color distribution of the ACS RGB may be a result of a superposition of stars in M101's outer disk with those in its halo. The ACS field is closer to the center of the galaxy than is the WFC3 field, such that any halo contribution could be greater in number or-in the presence of a halo metallicity gradient - redder in color (or both) than that shown in the WFC3 CMD. To assess the possible contribution of halo stars to the RGB in the ACS field, we examine three different scalings of the WFC3 populations. The first, most conservative, model simply takes the WFC3 CMD as observed, scaling up in number only to account for the different areas of the WFC3 and ACS fields. The second model shifts the WFC3 RGB redward in color by $0.11 \mathrm{mag}$ to match the mean color of the ACS RGB, and also scales it up in number by a factor of 2.27 , as might be expected if the halo surface density profile follows an $r^{-3}$ form consistent with the outer halos of MW, M31, and other spiral galaxies (e.g., Ibata et al. 2014; Harmsen et al. 2017; Medina et al. 2018 and references within). The third model keeps the $r^{-3}$ density scaling, but shifts the color redward by only $0.075 \mathrm{mag}$ to match the blue side of the ACS RGB color distribution. We note that either of the color shifts implies a strong metallicity gradient in the M101 halo. For example, for old (8-10 Gyr) stellar populations in the PARSEC 1.2S stellar isochrones (Bressan et al. 2012; Marigo et al. 2017; see below for details), a color shift of $0.11(0.075)$ mag over $11 \mathrm{kpc}$ would imply metallicity gradients of $\sim-0.035(-0.02)$ dex kpc ${ }^{-1}$, larger than observed in spiral galaxy halos to date (e.g., Gilbert et al. 2014; Monachesi et al. 2016; Harmsen et al. 2017).

Figure 7 shows the effect of subtracting these different halo RGB models from the observed color distribution of stars within 0.5 mag of the TRGB in the WFC3 field. Without any color shift or density scaling, the most conservative model would explain a large fraction of stars with colors intermediate between the RHeB stars and RGB stars as coming from the halo population, but would not significantly change the inferred color distribution of the ACS RGB itself. The model with the strongest color shift, scaled up by the $r^{-3}$ density scaling, provides a poor match to the observed ACS RGB due to its narrower color spread-the model matches the peak number of stars in the ACS RGB but cannot explain the broad spread in colors. The more modest color shift of the third model successfully matches the "blue wing" of the ACS RGB and would imply that about $2 / 3$ of the observed ACS RGB is due to a halo population seen in projection. Again, however, even this model implies a large metallicity gradient, and is thus likely to be an overestimate of the halo contamination. A more modest metallicity gradient would push any halo RGB contamination blueward and force a concurrent reduction in the number of halo RGB stars, so as not to overpredict the observed counts at these bluer colors. In summary, then, the RGB population seen in the ACS disk field appears to be only

\footnotetext{
5 While our use of the Jang \& Lee (2015) photometric transformation of WFC3 photometry to the ACS system has shifted the WFC3 RGB bluer by $\approx 0.04$ mag, this is much less than the $0.11 \mathrm{mag}$ difference we observe in the color of the RGB sequences in the two fields.
}

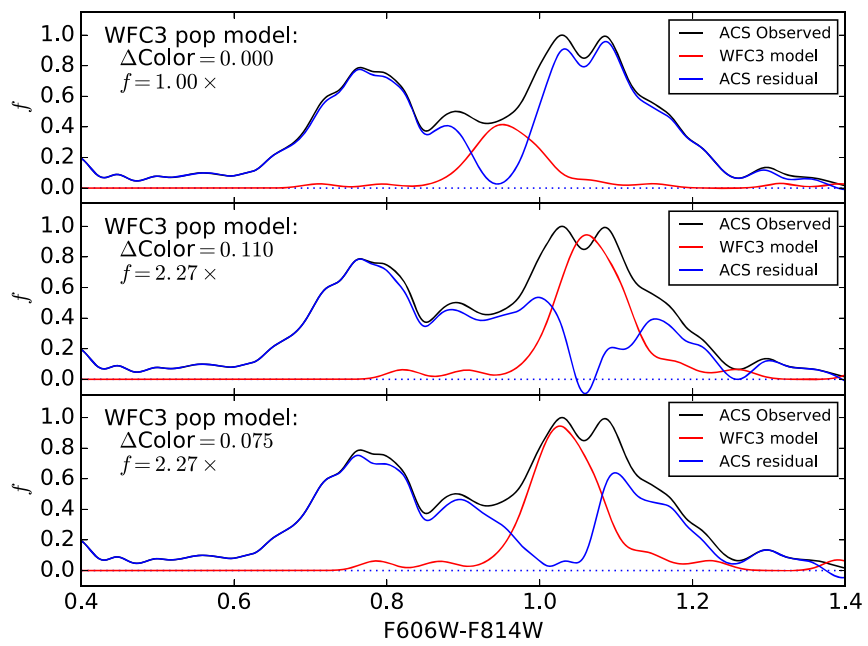

Figure 7. The effect of different halo population models on the color distribution of stars within 0.5 of the TRGB in the ACS disk field. See text for details.

modestly contaminated by halo RGB stars and must represent an in situ population of old stars in M101's outer stellar disk, distinct in metallicity from those in M101's stellar halo.

To gain a more quantitative understanding of the stellar populations in M101's outer regions, we compare the observed CMDs in the two fields to PARSEC 1.2S stellar isochrones ${ }^{6}$ (Bressan et al. 2012; Marigo et al. 2017) of varying age and metallicity. These isochrones use stellar models that presume scaled solar abundances and adopt $Z_{\odot}=0.0152$; in this paper, we refer to the model metallicities using the nomenclature $[M / H]=\log \left(Z / Z_{\odot}\right)$. In Figure 8, we concentrate on the RGB in the two fields, overlaying $10 \mathrm{Gyr}$ old isochrones with metallicities spanning the range $[M / H]=-2.0$ to -0.7 . In the WFC3 field, the RGB is bracketed reasonably well by the $[M / H]=-2.0$ and -1.5 isochrones. The RGB in the ACS field is slightly harder to trace, due to the proximity of the RHeB sequence; fainter than $M_{\mathrm{F} 814 \mathrm{~W}}=-2$, the two populations begin to blend together in the CMD. However, at brighter magnitudes, the redder and broader ACS RGB is bracketed by the $[M / H]=-1.7$ and -1.0 isochrones. We show this more directly in Figure 9, which shows the F606W - F814W color of the RGB (again measured as the average color within 0.5 mag of the TRGB) as a function of metallicity. For the isochrone age of $10 \mathrm{Gyr}$, the inferred mean metallicity for the WFC3 RGB is $[M / H]=-1.7 \pm 0.2$, and that for the ACS RGB is $[M / H]=-1.3 \pm 0.2$; modestly younger ages shift the inferred metallicity higher, but by only a small amountapproximately 0.2 dex for a $6.5 \mathrm{Gyr}$ old population.

\subsection{WFC3 Field: Surface Brightness}

To better compare our results to deep surface photometry of M101's integrated stellar light $(\mathrm{M}+13$, van Dokkum et al. 2014; Merritt et al. 2016), we can also calculate the expected surface brightness of the WFC3 field, given the observed RGB and AGB populations in the CMD. We start with the RGB, by summing the light from all stars detected along the RGB down to a cutoff magnitude of $\mathrm{F} 814 \mathrm{~W}=27.45$ $\left(M_{\mathrm{F} 814 \mathrm{~W}}=-1.75\right.$; see Figure 5$)$. This cutoff magnitude is just faint enough to allow us to sufficiently probe the RGB without

\footnotetext{
6 http://stev.oapd.inaf.it/cgi-bin/cmd
} 


\begin{tabular}{|l} 
Isochrone Age = $10 \mathrm{Gyr}$ \\
Isochrone [M/H]: \\
$-2.0 \quad-1.5 \quad-1 \quad-1.0$ \\
$-1.7 \quad-1.3 \quad-0.7$
\end{tabular}

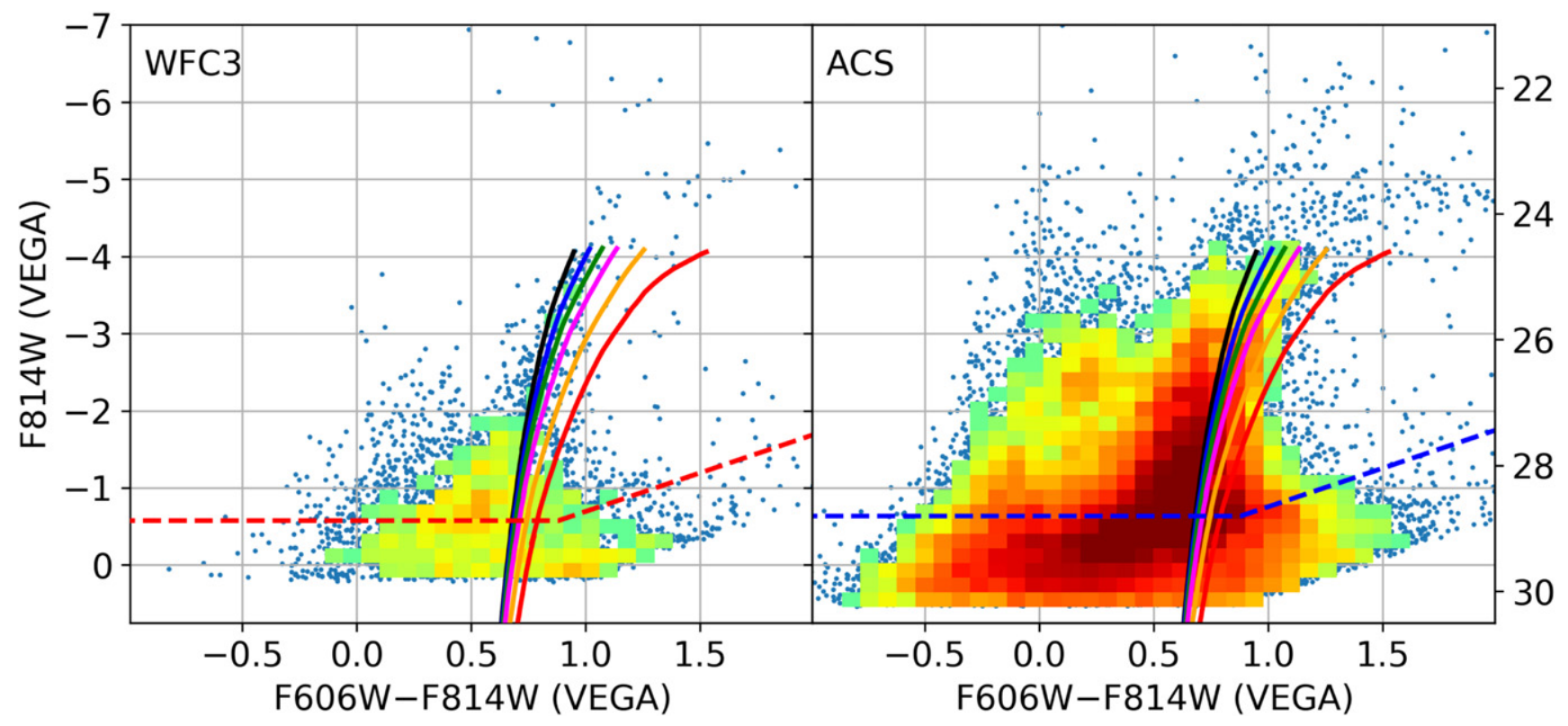

Figure 8. The WFC3 and ACS CMDs with $10 \mathrm{Gyr}$ old PARSEC 1.2S stellar isochrones (Marigo et al. 2017) overlaid.

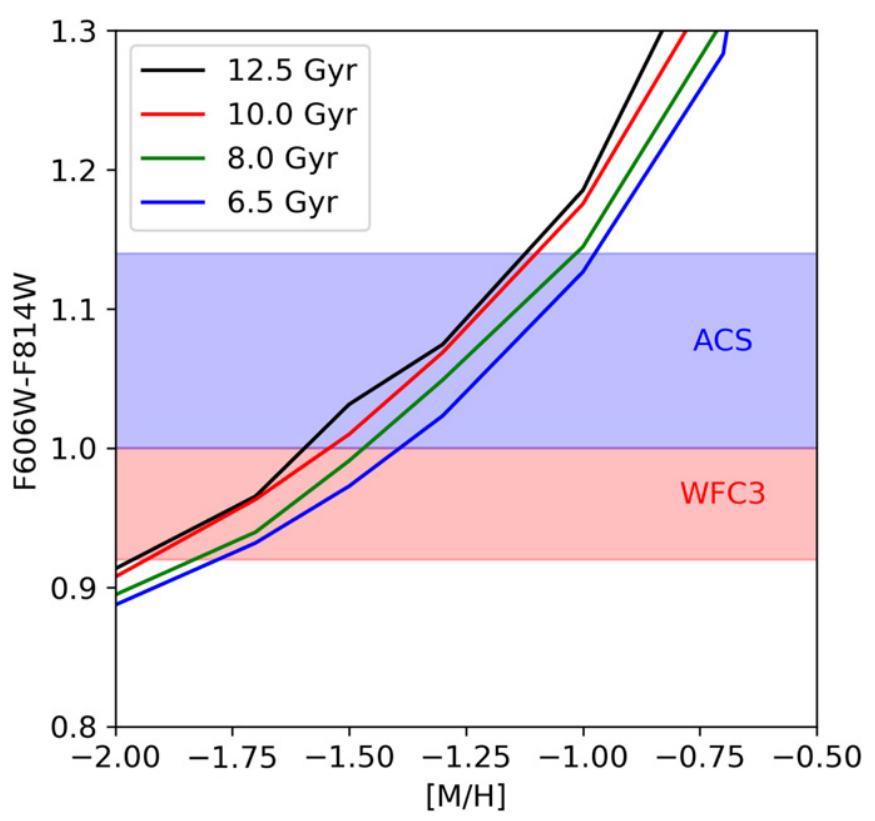

Figure 9. RGB color as a function of metallicity for PARSEC 1.2S isochrones (Marigo et al. 2017) with ages 6.5, 8, 10, and $12.5 \mathrm{Gyr}$. The red band shows the $\pm 1 \sigma$ color of the WFC3 RGB, while the blue band shows the color of the ACS RGB.

being dominated by the uncertainty due to background sources and incompleteness corrections at the fainter magnitudes. To remove background contamination, we select all objects within the same RGB selection region in the background Abell 2744 flanking field and subtract their summed flux from the estimate.
To calculate the total luminosity of the stellar population, we then use luminosity functions from the PARSEC $1.2 \mathrm{~S}$ models of Marigo et al. (2017) to calculate the "missing light fraction" $\left(f_{\mathrm{ML}}\right)$ from stars below our RGB cutoff magnitude. Given uncertainties in the inferred age and metallicity of the WFC3 RGB, we sample model luminosity functions that span a range of ages from 6 to 12 Gyr and metallicity $[M / H]=-1.7$ to -1.5 , deriving a missing light fraction of $f_{\mathrm{ML}, \mathrm{F} 814 \mathrm{~W}}=0.690 \pm 0.005$. After correcting for this missing light, we then add the combined luminosity from observed AGB stars, as the PARSEC models do not include any TP-AGB population in their estimate. To select the AGB population, we adopted the selection region as shown in Figure 5; we find 24 objects in the WFC3 CMD, compared to only four in the Abell 2844 background field. We then used the same methodology for the F606W surface brightness, except using a subset of the F814W RGB region that included all objects brighter than $\mathrm{F} 606 \mathrm{~W}=27.89\left(M_{\mathrm{F} 606 \mathrm{~W}}=-1.30\right)$. For this cutoff, the fraction of missing $\mathrm{F} 606 \mathrm{~W}$ from fainter stars is $f_{\mathrm{ML}, \mathrm{F} 606 \mathrm{~W}}=0.780 \pm 0.005$, again ignoring the $\mathrm{AGB}$ contribution.

After applying the model corrections and adding the (observed) AGB component to our RGB luminosities, we derive a total $\mu_{\mathrm{F} 814 \mathrm{~W}}=30.02 \pm 0.05 \mathrm{mag}_{\operatorname{arcsec}^{-2}}$, where the error is based on bootstrap resampling of the $F 814 W$ luminosities for all stars within the RGB and AGB selection regions, along with the uncertainty in the missing light correction. Similarly, we find $\mu_{\mathrm{F} 606 \mathrm{~W}}=30.74 \pm 0.05 \mathrm{mag} \operatorname{arcsec}^{-2}$ for our WFC3 field. It is important to note that these surface brightness results are quite robust to systematic uncertainties in the background contamination. Assuming a (generously) large $\pm 30 \%$ error in our absolute background subtraction yields an additional shift of \pm 0.05 in any of the above results. 
To facilitate comparison with previous surface photometry, we convert the F606W and F814W magnitudes to $V$ and $I$ using the observed Sirianni et al. (2005) transformations, yielding $\mu_{I}=29.99 \pm 0.05 \mathrm{mag} \operatorname{arcsec}^{-2}$ and $\mu_{V}=$ $30.86 \pm 0.05 \mathrm{mag} \operatorname{arcsec}^{-2}$. These values are fainter than the $\mu_{V}=29.0 \mathrm{mag} \operatorname{arcsec}^{-2}$ surface brightness limit of the Mihos et al. (2013) surface photometry, consistent with the non-detection of any diffuse light in this particular region. The resulting color of the M101 stellar population is $V-I=0.87 \pm 0.07$ for the entire field, a value consistent with the colors of metal-poor Milky Way globular clusters (e.g., Harris 1996, 2010), as well as with the metallicity of the RGB stars detected in this field. Finally, to compare our surface brightnesses to the deep $g$-band surface photometry of van Dokkum et al. (2014), we convert our $\mu_{V}$ value using transformations from Jordi et al. (2005) for metal-poor stars. To make use of their $V$ to $g$ transformation, we first adopt a color $B-V=0.65 \pm 0.05$, derived using the $B-V, V-I$ colors of Milky Way globular clusters (e.g., Harris 2010). We thus derive $\mu_{g}=31.10 \pm 0.07 \mathrm{mag} \operatorname{arcsec}^{-2}$, where the error is solely from the uncertainties in our derived surface brightnesses, and not from any color transformation errors. This derived surface brightness is $\sim 1-2 \mathrm{mag} \operatorname{arcsec}^{-2}$ brighter than the azimuthally averaged surface brightness profile of M101 at this radius shown in van Dokkum et al. (2014) and Merritt et al. (2016), a sign perhaps either of azimuthal substructure in M101's halo or systematic uncertainties in the broadband surface photometry. We comment further on the comparison with broadband imaging in the Discussion.

\subsection{Young Stellar Populations in the ACS Disk Field}

The young stellar populations seen in the ACS disk field give us an opportunity to use the the red and blue heliumburning sequences to probe the age and metallicities of those stars as well (see, e.g., McQuinn et al. 2011). As massive stars evolve back and forth across the CMD on their "blue loops," they pile up in the CMD near the turnaround spot on their evolutionary tracks. Roughly speaking, the BHeB and RHeB sequences thus trace out these turnaround points in the track as a function of stellar mass or age-the more luminous stars on the sequences are more massive and younger than stars further down the sequences. Because the turnaround spots on the tracks are also a strong function of stellar metallicity, we can use the sequences to probe age and metallicity of the young populations in the field.

Figure 10 overlays younger PARSEC isochrones for metallicities in the range $[M / H]=-1.7$ to -0.3 . The match to blue and red helium-burning sequences is of particular interest here. At the lowest metallicities shown $([M / H] \leqslant-1.7)$, the model isochrones place both the red and blue helium-burning tracks too blue to match the observed sequences well. The helium-burning tracks at magnitudes brighter than $M_{\mathrm{F} 814 \mathrm{~W}}=-3$ (stellar ages $<100 \mathrm{Myr}$ ) reach colors $\mathrm{F} 606 \mathrm{~W}-\mathrm{F} 814 \mathrm{~W}<0.0$, while the observed $\mathrm{BHeB}$ spans a range of color $0.0<\mathrm{F} 606 \mathrm{~W}-\mathrm{F} 814 \mathrm{~W}<0.25$. Similarly, at these metallicities, the helium-burning tracks brighter than $M_{\mathrm{F} 814 \mathrm{~W}}=-4$ never become redder than $\mathrm{F} 606 \mathrm{~W}-$ $\mathrm{F} 814 \mathrm{~W}=1.0$, whereas the brightest part of the observed RHeB sequence reaches colors of F606W $-\mathrm{F} 814 \mathrm{~W}=1.3-1.4$. Conversely, at the highest metallicities $([M / H] \geqslant-0.7)$, the heliumburning tracks never extend blue enough to explain the bulk of the observed $\mathrm{BHeB}$ sequence. On the red side of the metal-rich tracks, the most massive stars (ages $<200 \mathrm{Myr}$ ) do fall on the observed RHeB sequence, but metallicities this high cannot then match the handful of very luminous stars at $M_{\mathrm{F} 814 \mathrm{~W}}<-6$, F606W - F814W $\sim 1.0$ that are explained in the lower metallicity tracks as the youngest, most massive RHeB stars. Furthermore, at these high metallicities, the model tracks for stars $\sim 300-500$ Myr old would fill in the region between the observed RHeB and RGB populations, while the CMD itself shows good separation between those populations.

In contrast, most features in the observed CMD are wellreproduced by metallicities in the range $-1.3 \leqslant$ $[M / H] \leqslant-1.0$. On both the blue and red sides of the heliumburning tracks, the turnaround colors track the observed $\mathrm{BHeB}$ and $\mathrm{RHeB}$ sequences, while the brightest stars (at $M_{\mathrm{F} 814 \mathrm{~W}}<-6, \mathrm{~F} 606 \mathrm{~W}-\mathrm{F} 814 \mathrm{~W} \sim 1.0$ ) are explained by a small number of young, high-mass stars in the field. This last population, a sign of very recent or ongoing star formation, is also seen as a smattering of stars still on the main sequence with $M_{\mathrm{F} 814 \mathrm{~W}}>-4$ and F606W $-\mathrm{F} 814 \mathrm{~W}<-0.1$. Based on the good qualitative match of these isochrones to the young populations in the CMD, we adopt a final metallicity estimate of $[M / H]=-1.15 \pm 0.15$, where the uncertainty estimate simply reflects the range of isochrones that match the data reasonably well.

The inferred metallicity of the young population provides an interesting comparison to that of the older RGB population discussed in Section 3. While the young isochrones shown in Figure 10 permit metallicities as high as $[M / H]=-1.0$, such a metallicity makes for a poor match to the RGB sequence. The mean metallicity inferred for the old RGB population in the ACS field is lower by $\sim 0.15 \mathrm{dex}$, compared to the young populations, suggesting that we may be seeing evidence for chemical evolution in M101's outer disk. However, this metallicity difference is small and likely at the limit of our ability to discriminate metallicity differences, given possible age spreads in the old population as well as uncertainties in the isochrones and stellar population models.

The helium-burning sequences also deliver information regarding the detailed history of recent star formation in the field. Along the $\mathrm{BHeB}$ sequence, the distribution of stellar luminosity is a function of the star formation history, IMF, and evolutionary rate of stars along the helium-burning tracks. For constant (or slowly varying) star formation rates, the number of stars should drop smoothly at increasing luminosity along the sequence, simply reflecting the declining numbers of stars at high mass and their faster rates of evolution. Such behavior can be seen in a variety of CMDs of star-forming dwarf galaxies in the local group (McQuinn et al. 2011). However, this is not what we observe in the ACS field, which shows a discrete "lump" of stars" in the $\mathrm{BHeB}$ sequence at $M_{\mathrm{F} 814 \mathrm{~W}} \approx-2.4$, $\mathrm{F} 606 \mathrm{~W}-\mathrm{F} 814 \mathrm{~W} \approx 0.22$, rather than a monotonically decreasing number of stars along the sequence.

This BHeB lump is likely a cohort of stars with common age and metallicity evolving through the blue loop phase. A comparison with the isochrones in Figure 10 shows that, for stars in the metallicity range -1.3 to -1.0 , the lump corresponds to stellar ages of $300-400 \mathrm{Myr}$, suggesting this region of M101's outer disk experienced a weak burst of star formation $\sim 350 \mathrm{Myr}$ ago. This age is similar to the starburst

\footnotetext{
7 We refer to this stellar population as "lump stars," in keeping with the astronomical tradition of using names that end in "-ump" to refer to piles of stars in CMDs - e.g., red clump or AGB bump stars.
} 


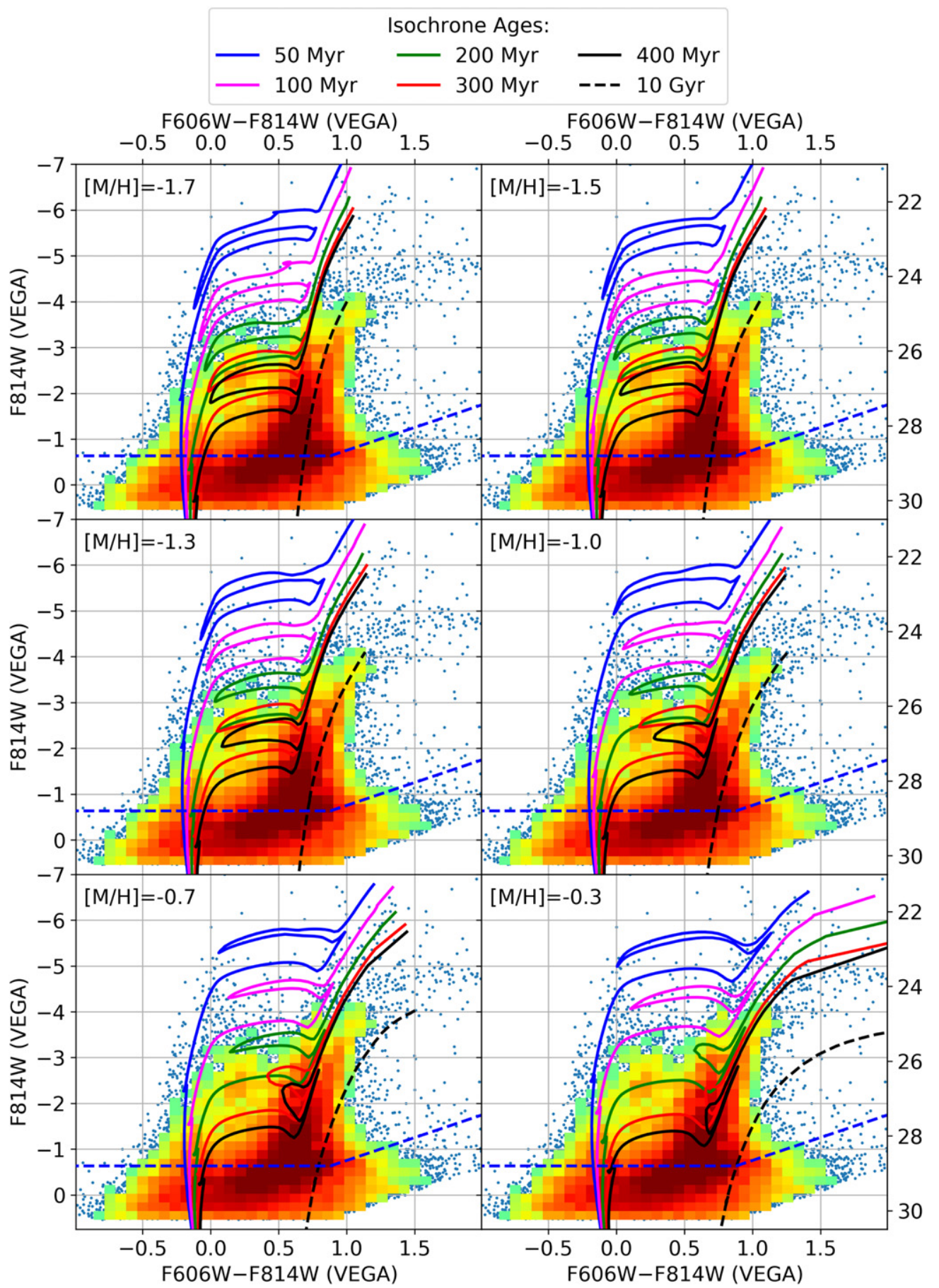

Figure 10. Young stellar isochrones (PARSEC 1.2S; Marigo et al. 2017) overlaid on the ACS CMD. Each panel shows isochrones of varying ages for a given metallicity.

age inferred in $\mathrm{M}+13$, where the very blue colors of the integrated starlight, coupled with the lack of significant FUV emission in the NE Plume, also argued for a weak outer disk starburst of age 250-350 Myr. The slightly younger age inferred from the broad band colors is likely due to the fact that $\mathrm{M}+13$ focused on the integrated properties of the NE Plume as a whole, whereas our ACS imaging targets a low surface brightness portion of the Plume that is preferentially redder and avoids the Plume's most active star-forming environments. It is not surprising, therefore, that we see populations that are slightly older than those inferred for the Plume as a whole.

While the isochrones shown in Figure 10 show the positions of the various stellar population sequences in the CMD, they do not give information on the relative numbers of stars populating those sequences. As a result, they contain little information on the age distribution of the young population. In contrast, star counts along the $\mathrm{BHeB}$ sequence-and in particular, the compactness of the $\mathrm{BHeB}$ lump-are related to the age spread in the evolving cohort of young stars, with a 
narrow age spread leading to a more compact lump. To constrain the duration of the outer disk starburst, we use the synthetic CMD modeling package IACstar (Aparicio \& Gallart 2004) to model the effect of the burst duration on the structure of the observed BHeB lump. As a secondary goal, the IACstar modeling gives a consistency check on the ages and metallicities inferred from the isochrone matching shown in Figures 8 and 10.

To construct a synthetic CMD for comparison to observations, the IACstar package uses the input star formation history, metallicity model, and stellar IMF to populate a CMD for a given choice of stellar evolutionary library. We again characterize metallicity as $[M / H]=\log \left(Z / Z_{\odot}\right)$, although we note that IACstar adopts $Z_{\odot}=0.02$, which introduces a slight 0.1 dex shift in the $[M / H]$ values when compared to those inferred from the PARSEC isochrones, which adopted $Z_{\odot}=0.0152$. This shift is likely less than the overall uncertainty in the CMD modeling, so for consistency of comparison, we place the IACstar models on the same solar metallicity zero point as the PARSEC models by setting $Z_{\odot}=0.0152$ when converting metallicity from $Z$ to $[M / H]$. Finally, when modeling the CMDs, we adopt a Kroupa (2001) IMF along with the Teramo stellar libraries (Pietrinferni et al. 2004).

We start by generating model CMDs for a variety of burst populations, modeling the star formation histories as Gaussian in time. These Gaussian bursts peak at a time $t_{\text {burst }}$ in the past, with a duration given by $\sigma_{b}$ and a metallicity $[M / H]$. In modeling the synthetic CMDs with IACstar, we found the models best match the location of stars in the lump and RHeB sequence with a metallicity of $[M / H]=-1.25$, slightly lower than that inferred from the PARSEC isochrones $([M / H]=-1.15)$, even after adjusting for the differing solar metallicity zeropoints. We thus designate our fiducial model as one with burst age $t_{\text {burst }}=300 \mathrm{Myr}$, burst duration $\sigma_{b}=50 \mathrm{Myr}$, and burst metallicity $[M / H]=-1.25$, then vary the burst parameters around this fiducial model to explore the effect of burst age, burst spread, and metallicity on the structure within the helium-burning sequences. To construct the model CMDs, we begin by using IACstar to generate a CMD for each model with 100,000 synthesized stars brighter than $M_{V}=+1.3$, significantly fainter than our $50 \%$ completeness limit. We turn the absolute magnitudes into observed apparent magnitudes using our adopted M101 distance modulus of $(m-M)_{0}=29.19$, and add foreground extinction from Schlafly \& Finkbeiner (2011). We then add noise to the synthetic CMD using the artificial star tests from Section 2. In short, for each model star, we match it to the artificial star closest in color and magnitude, and give it the photometric error found in that artificial star. In the case where the matched artificial star was not recovered in the artificial star test, we delete the CMD star from the synthesized CMD. In this way, we match the synthesized CMD to the observed CMD in both photometric uncertainty and completeness. Finally, we downsample the total number of stars in the synthesized CMD, such that it has the same number of stars in the region of the $\mathrm{BHeB}$ lump as are observed in the real data. For this exercise, we do not add background contamination, because we are interested in the intrinsic structure of the young populations in the CMD.

Figure 11 shows the resulting model CMD for these various starburst scenarios. In each panel, the location of the $\mathrm{BHeB}$ lump in the observed ACS CMD is shown by the black box, while a parallelogram marks the spine of the RHeB sequence (with no old stars in these burst models, there are no RGB or AGB populations). The top row of the figure shows the results of varying the burst age. It can be clearly seen that varying $t_{\text {burst }}$ by $\pm 100 \mathrm{Myr}$ moves the lump in the synthetic CMD significantly away from the observed region; more recent bursts show lumps that are brighter and somewhat bluer than the observed lump, while older bursts are systematically redder and fainter. The middle row shows the effect of changing metallicity; at $[M / H]=-1.6$, the $\mathrm{BHeB}$ lump is too blue, while at $[M / H]=-0.9$, both the BHeB lump and the RHeB spine have moved too red. Finally, the bottom row shows changing burst duration. With a very highly synchronized burst of $\sigma_{b}=25 \mathrm{Myr}$, the $\mathrm{BHeB}$ population shows a very narrow range of absolute magnitude, and the lump is actually too concentrated, with no stars along the broader BHeB sequence. As the burst duration is increased to $\sigma_{b}=100 \mathrm{Myr}$, stars begin to populate a broader range of the $\mathrm{BHeB}$ sequence, but the discrete lump in the CMD is smeared out, making it a qualitatively poor match to the observed CMD. These models thus suggest a reasonable estimate for the burst duration to be $\sigma_{b}<100$ Myr.

Fully modeling the observed CMD requires a detailed reconstruction of the star formation and chemical evolution histories behind the multiple stellar populations seen in the field: the older RGB stars, the post-starburst population seen in the $\mathrm{BHeB}$, and the younger stars from ongoing star formation, as well as the population of halo stars projected onto the field. Such a reconstruction is beyond the scope of our present work; instead, we compare the observed CMD to ones modeled using a simple "disk plus burst" star formation history. The disk population is modeled using a constant star formation rate over the past $12 \mathrm{Gyr}$, and gives rise to the old and intermediate-age populations seen in the CMD. For simplicity, we use a fixed metallicity of $[M / H]=-1.25$ for the disk population, based on the best-matching isochrones shown for the old population in Figure 10. The fiducial starburst population is then characterized by a Gaussian with $t_{\text {burst }}=300 \mathrm{Myr}$, burst duration $\sigma_{b}=50 \mathrm{Myr}$, and metallicity $[M / H]=-1.25$, based on the best matches shown in Figure 11, although we also run a series of starburst models with varying post-burst behavior (see below). We add observational noise to the populations as described previously, and then combine the populations. We set the relative mass fraction of the disk and burst populations by matching the relative numbers of stars observed on the RGB (brighter than $M_{\mathrm{F} 814 \mathrm{~W}}=-1.75$; fainter than this, the RGB and RHeB begin to overlap) to those found in the BHeB lump $\left(N_{\mathrm{RGB}} / N_{\text {lump }}=3.17\right)$. For the models considered here, the burst fraction $f_{b}=M_{\text {burst }} / M_{\text {disk }}$ ranges from $6.5 \%$ to $14.5 \%$, depending on the details of the burst history (see Table 1).

In addition to these model populations, however, we must add an appropriate background population as well, accounting both for unresolved background sources and for the presence of M101 halo stars projected onto the M101 disk. For this, we adopt the WFC3 halo field CMD as the appropriate background, because it includes both true background contamination plus any contamination from M101's stellar halo. To account for the different FOV of the ACS and WFC3 cameras, we scale up the WFC3 counts by a factor of 1.55 (the relative area of ACS versus WFC3) via random duplication of WFC3 sources after adding a \pm 0.05 mag scatter to their magnitudes. We again note, however, that this approach implicitly assumes 

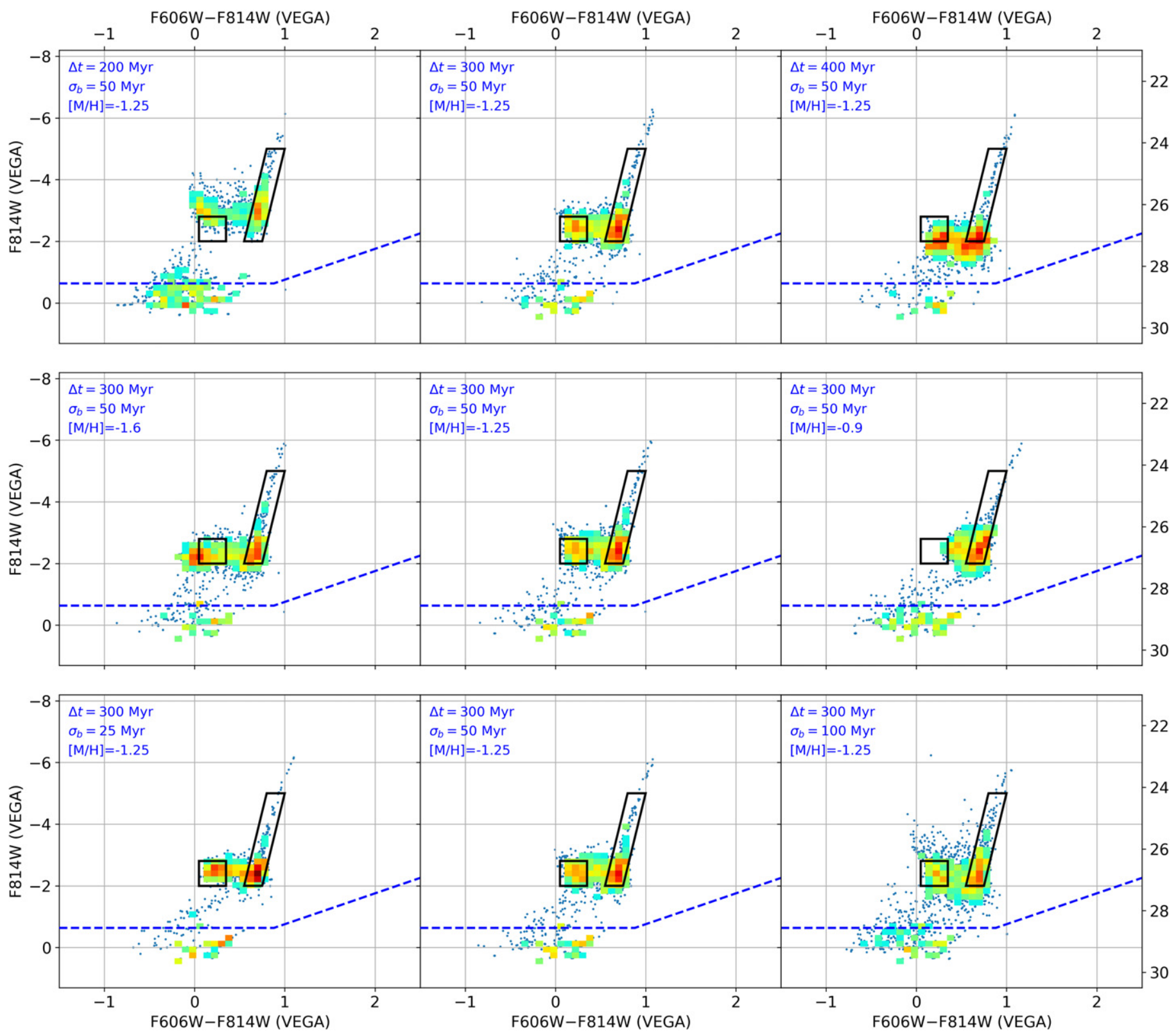

Figure 11. IACstar model CMDs for young starburst populations of different metallicity. In each case, the star formation history of the model is given by a Gaussian that peaked at a time $\Delta t$ in the past and had a width $\sigma_{b}$. The top row varies the burst age $\Delta t$, the middle row varies the burst metallicity [M/H], and the bottom row varies the burst duration $\sigma_{b}$. The boxes show the positions of the observed BHeB lump and RHeB sequence in the outer disk CMD.

that the halo population found in the WFC3 field is identical in number density to the one that contaminates the ACS fieldtherefore, it may well underestimate the true halo population in the synthesized CMDs, as discussed previously.

Figure 12 shows both the observed ACS CMD and the modeled CMDs for these various composite star formation histories, while Table 1 details the population number counts (relative to the observed numbers) and integrated light properties for each modeled CMD. The fiducial pure Gaussian model is shown top center, and it does a reasonable job of matching the observed CMD, with one notable exception: it has significantly fewer main sequence stars than observed, particularly those brighter than $M_{\mathrm{F} 814 \mathrm{~W}}=-2$. Such luminous main sequence stars must have ages $\lesssim 200 \mathrm{Myr}$ (see, e.g., Figure 10), arguing that there must be residual ongoing star formation above that predicted by the simulated star formation history. This is also borne out by the broadband color of the integrated light in the model (shown in Table 1), which is somewhat too red compared to the broadband $B-V$ colors in the field derived from the deep imaging of $\mathrm{M}+13$.

To examine the effects of late-time residual star formation, we look at three variants on the Gaussian burst model: one where, after ramping up, the SFR stays constant at the peak level to the present day ("continuing burst"), and two where the SFR declines linearly to half-peak ("half-faded") or zero amplitude ("fully faded") at present. $^{8}$ These model star

\footnotetext{
8 This does not mean there is no star formation at present; all models continue to have ongoing star formation from the constant SFR "disk" component; see the SFR histories in Figure 12. Note also that all models are consistent with the upper limit on current star formation rate in the ACS field $\left(\approx 0.002 M_{\odot} \mathrm{yr}^{-1}\right)$ derived from the $\mathrm{H} \alpha$ imaging of Watkins et al. (2017).
} 
Table 1

Composite IACstar Models

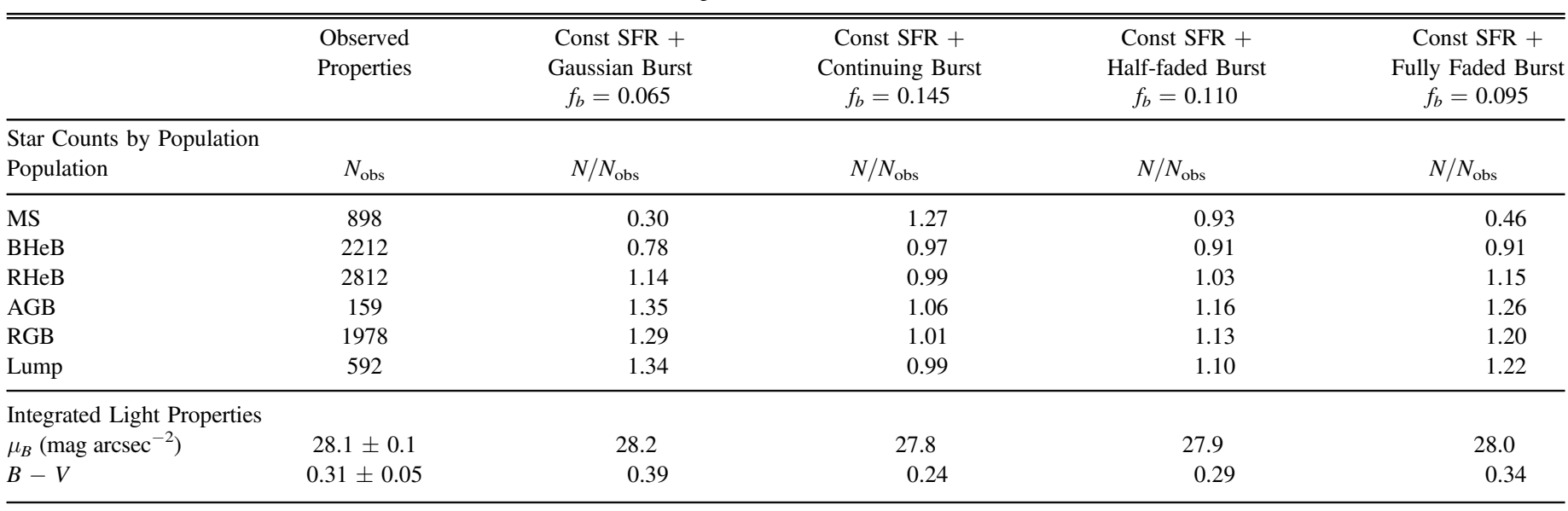

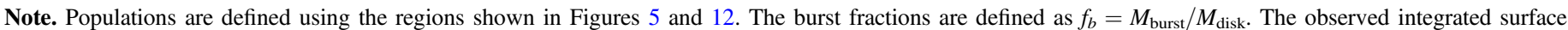
brightness and color of the field come from the imaging of Mihos et al. (2013).

formation histories are shown in the upper right panel of Figure 12, and the resulting CMDs are shown in the bottom panels. The continuing burst model now somewhat overpredicts the MS population and yields integrated colors that are slightly too blue, and the $\mathrm{BHeB}$ sequence in the CMD appears much more "stream-like" than in the observed CMD. In the half-faded burst model, the BHeB shows more of the discrete lump seen in the data, in addition to more closely matching both the number counts and broadband colors of the region. In contrast, the fully faded burst model significantly underpredicts the MS population, resulting in a poorer match to the data. Clearly, these star formation histories are simple cartoon models of the detailed history of star formation in M101's outer disk. Nonetheless, the modeled CMDs corroborate the inference, via isochrone matching, that the stellar populations trace a recent $\sim 300$ Myr old weak burst of star formation in the outer disk, which has partially but not completely faded at the present day.

\subsection{Spatial Distribution of Stellar Populations}

We can also use the CMDs to trace the spatial distribution of different stellar populations across the ACS field of view. While we reserve a quantitative study of spatial clustering for a future paper, we present here a broad comparison of the spatial distribution of the stellar populations to the broadband diffuse light. In the top left panel of Figure 13, we show the populations defined on the CMD: main sequence (MS), blue and red helium-burning sequences (BHeB, RHeB), the co-evol lump stars in the $\mathrm{BHeB}$ (lump), and stars on the red giant and AGBs (RGB, AGB). We then plot the location of stars in each stellar population, along with the deep B-band imaging of $\mathrm{M}$ +13 , masked of bright, compact sources and median binned to $13^{\prime \prime}$ resolution, in order to show low surface brightness structure. A comparison of all stellar sources brighter than $\mathrm{F} 814 \mathrm{~W}=28.56$ (the $50 \%$ completeness limit of the ACS field) shows a good match to the broadband imaging - the highest densities of stars are found in the north and south corners of the field and along the NW edge, regions that correspond to a higher integrated surface brightness of blue starlight. The population of main sequence stars follows a similar spatial distribution and shows significant clustering; at these luminosities $\left(M_{\mathrm{F} 814 \mathrm{~W}}<-1\right)$, the main sequence lifetime is short (less than $100 \mathrm{Myr}$ ), such that these stars have not had time to drift far from their birthplaces. The more evolved helium-burning sequences, which cover a larger age spread (up to $500 \mathrm{Myr}$ ), are not as clustered and are weaker tracers of the broadband blue light. In contrast, the older RGB stars (ages $>1$ Gyr) are more smoothly spread across the field, and if anything, they show a slight excess in the eastern (left) side of the image, unlike the blue light. While the blue light and massive young stars together follow the spatial distribution of recent star formation, the RGB stars may be tracing the underlying mass density of M101's outer disk. Interestingly, sources in the BHeB lump, which should consist of a coevolutionary population with age $\sim 300 \mathrm{Myr}$, seem distributed more like the RGB stars on larger scales, although we do also see signs of small-scale clustering in this population as well. The spatial disconnect between the lump stars and the blue light implies that the spatial pattern of star formation across the field has changed over this $300 \mathrm{Myr}$ timescale, or that stars in the $\mathrm{BHeB}$ lump have drifted far from their original birthsites. The latter scenario is not unlikely; if young stellar populations have velocity dispersions of $\sigma_{v} \sim 10-20 \mathrm{~km} \mathrm{~s}^{-1}$, similar to that of young stellar populations in the Milky Way (e.g., Dehnen \& Binney 1998), then over a $300 \mathrm{Myr}$ span, they could have drifted roughly $\sigma_{v} \times t \approx 3-6 \mathrm{kpc}$, which is comparable in scale to the $\approx 7 \mathrm{kpc}$ size of the ACS field of view.

\section{Discussion}

Our HST imaging of fields in the outskirts of M101 provides new information on the galaxy's star-forming history and the properties of stellar populations in its outer disk and halo. In the ACS disk field, we see a metal-poor population consisting of both old red giant stars and young massive stars, and confirm the post-starburst nature of the populations suggested by the broadband imaging of $\mathrm{M}+13$. In the WFC3 halo field, we see only older populations-RGB and AGB stars-with much lower metallicities than the disk populations observed in the ACS field. In this section, we now turn to several questions that remain open: the metallicity of the outer disk, the origin of the stars in the WFC3 field, the nature of the detected AGB populations, and the properties of M101's halo compared to 


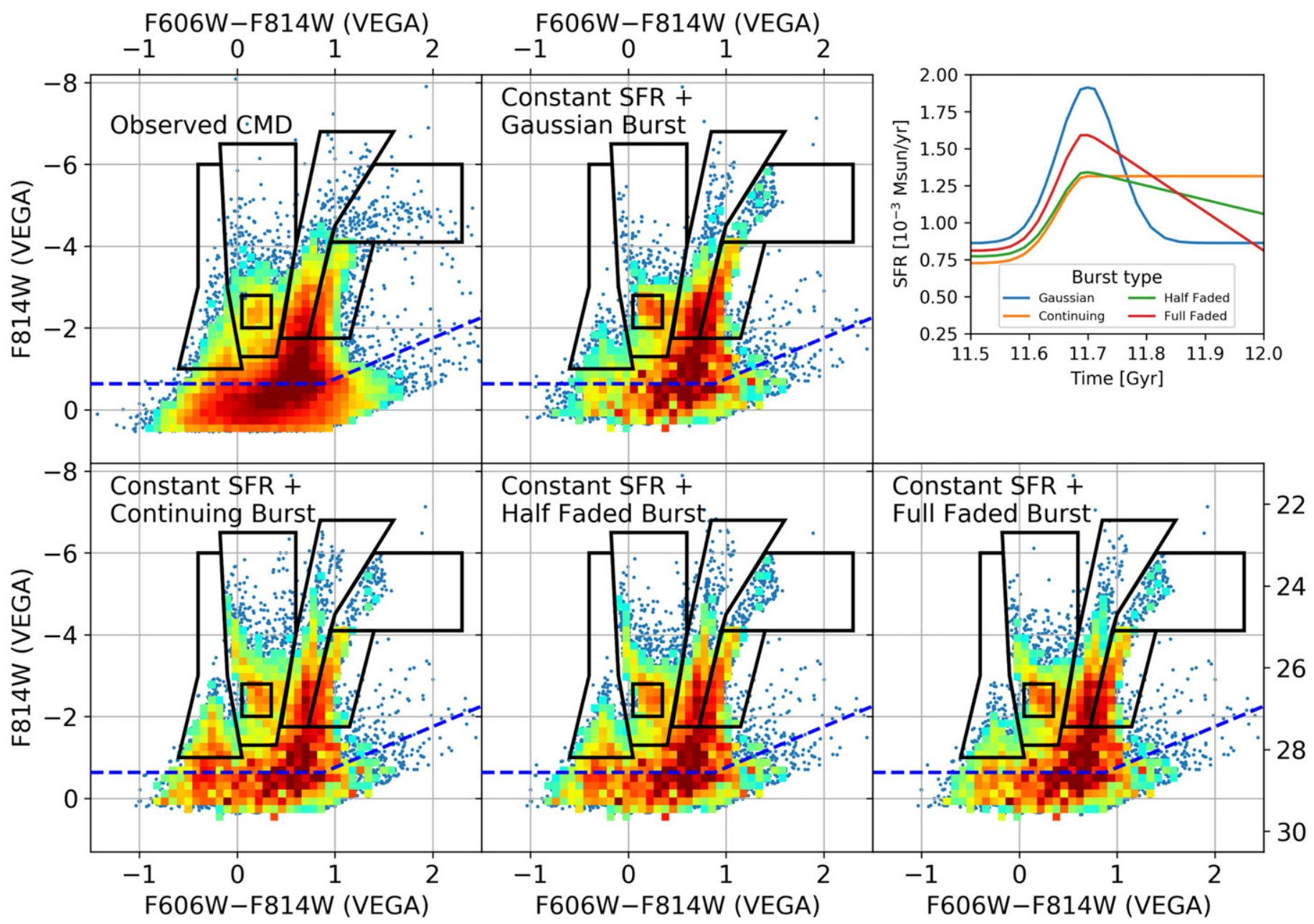

Figure 12. IACstar model CMDs for composite stellar populations, consisting of a constant star formation history plus recent bursts of varying star formation histories. The top row shows the observed CMD and the fiducial Gaussian burst model, while the bottom row shows three variants of the model where the late time history of the burst is varied. The star formation models are shown in the inset panel in the top row. All CMDs are modeled using a fixed metallicity of $[M / H]=-1.25$. See text for details.

those of other galaxies. We then present a cross-comparison between the resolved stellar population work presented here and the inferences from deep broadband imaging of M101's integrated light. Finally, we frame our results in the overall context of M101's dynamical history of interactions within the group environment.

\subsection{The Metallicity of the Outer Disk}

The metallicities we measure for the stellar populations in the outer disk are extremely low $([M / H]<-1)$, motivating a comparison with metallicities inferred in the outskirts of other disk galaxies. In performing this comparison, we note that the IACstar models give a slightly lower metallicity (by $\sim 0.1 \mathrm{dex}$ ) than the PARSEC isochrones suggest. Again, this difference is likely within the uncertainties of the models, and indeed we note that, characterized by metal abundance $Z$, the two techniques agree: the PARSEC isochrones that bracket the young populations in metallicity are $Z=0.00076$ and $Z=0.00152$, while the best match IACstar models use $Z=0.0008$. On balance, we favor the PARSEC models due to their use of more recent stellar models, and thus adopt a value of $[M / H]=-1.15 \pm 0.2$ as our best-match metallicity for the young populations in M101's outer disk, and a slightly more metal-poor $[M / H]=-1.3 \pm 0.2$ for the older RGB stars.
The quoted error bars simply reflect the range of isochrone metallicities that bracket the observed populations shown in Figures 8 and 10 .

Metallicity gradients in spiral galaxies can be most easily traced through H II region abundances. These gradients typically follow an exponential decline with radius, but then often flatten in the disk outskirts, such that extremely low metallicities are uncommon; see, e.g., the review by Bresolin (2017). For example, in M83, there appears to be a metallicity "floor" of $12+\log \mathrm{O} / \mathrm{H} \simeq 8.4$ (Bresolin et al. 2009); for a solar oxygen abundance of $12+\log \mathrm{O} / \mathrm{H}=8.69$ (Asplund et al. 2009), and assuming scaled solar abundances, this corresponds to $[M / H] \simeq-0.3$. In the Milky Way, both young star clusters and Cepheid variables also show evidence for a flattened metallicity gradient beyond $12 \mathrm{kpc}$, with typical outer disk metallicities of $[M / H] \sim-0.3$ (e.g., Luck et al. 2003; Yong et al. 2005; Sestito et al. 2008; Pedicelli et al. 2009). Compared to these values, our inferred metallicity for the young populations $([M / H]=-1.15)$ is significantly lower. However, in M101 itself, the H II region oxygen abundances show no clear break from a pure exponential (Kennicutt et al. 2003; Croxall et al. 2016), and the outermost disk H II regions show metallicities of $12+\log \mathrm{O} / \mathrm{H}=7.55$ (Kennicutt et al. 2003), equivalent to $[M / H]=-1.14$, in quite good 

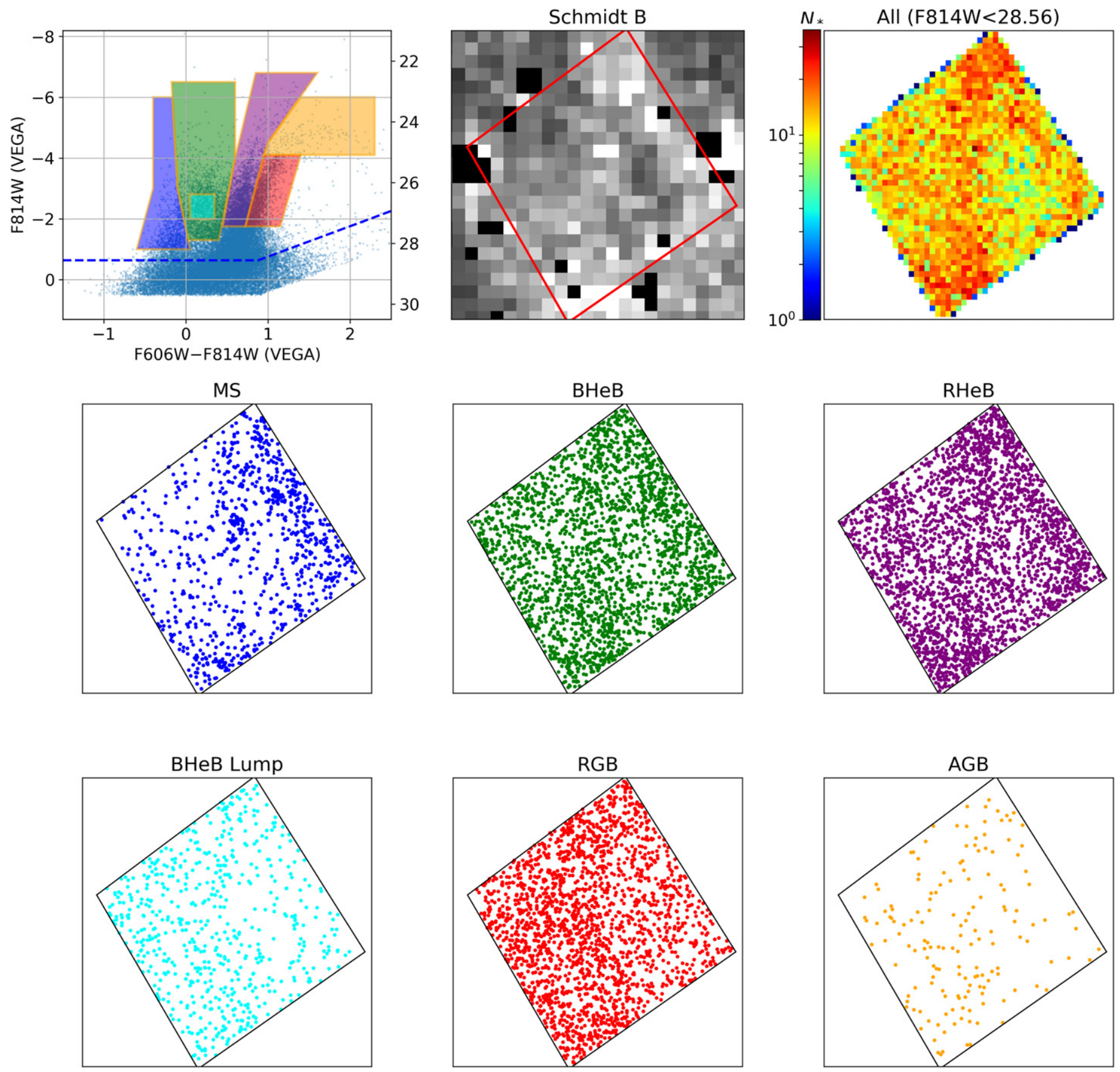

Figure 13. The spatial distribution of stars in the ACS field. The top left panel shows the CMD, with colored regions associated with varying stellar populations. The top middle panel shows the broadband B image from the Burrell Schmidt telescope $(\mathrm{M}+13)$, masked and rebinned to show low surface brightness structure, while the top right panel shows the spatial distribution of all stars brighter than the $50 \%$ completeness limit $(\mathrm{F} 814 \mathrm{~W}=28.56)$ in the ACS field. Subsequent panels show the spatial distributions of main sequence stars (MS), blue and red helium-burning stars (BHeB and RHeB), stars in the BHeB lump, and red giant and asymptotic giant branch stars (RGB and AGB).

agreement with our inferred metallicity for the young population. This match between the gas-phase metallicity and that of the stellar populations we see in the field is strong evidence that these populations were formed largely in situ rather than being scattered or migrating populations from the more metal-rich inner disk.

Metallicities for old stellar populations in disk outskirts are harder to come by. The low surface brightness of these regions makes all but the nearest disk galaxies difficult to study by means other than integrated colors, which suffer from the wellknown age-metallicity degeneracy and thus provide only the crudest of information on stellar metallicities. Instead, resolved population studies have largely been confined to a relatively few disk galaxies found within a few Mpc of the Milky Way. In M31, for example, studies of resolved RGB stars show that the metallicity gradient in the disk flattens at large radius; beyond $20 \mathrm{kpc}$, the typical metallicities are $[M / H] \sim-0.5$ (e.g.,, Worthey et al. 2005; Bernard et al. 2015), significantly higher than what we find for M101. In contrast, while the lowerluminosity Sculptor Group spirals NGC 300 and NGC 7793 also show flat outer disk metallicity profiles, their outskirts show much lower metallicities of $[M / H] \simeq-1.0$ and -1.5 , 
respectively (Vlajić et al. 2009, 2011). The metallicities we infer for the old populations in the ACS field $([M / H]=-1.3 \pm 0.2)$ are similarly low.

\subsection{The Origin of Stars in the WFC3 Field}

We targeted the WFC3 camera in a blank field away from the observed stellar disk in order to sample populations in M101's stellar halo. However, one concern with the interpretation of the WFC3 field as a halo field is its proximity to M101's distorted outer disk. Projected only a few kpc away from the extended NE Plume, the stars we detect in the WFC3 field could, in principle, be an extension of M101's disk to an even larger radius, or a population of stars scattered outward from M101's disk due to interactions between M101 and its companions.

However, the mix of stellar populations in the two fields is quite different, with no sign of a young population in the WFC3 field beyond those sources consistent with background contamination. If the populations in the ACS and WFC3 fields were similar, the helium-burning sequences should be visible in the WFC3 CMD, even accounting for its smaller number of stars. We see no such sequences, and the smattering of blue stars seen in the CMD is consistent with that expected from background contamination (see Figure 4). Based on the lack of a young population alone, the WFC3 field is not simply an extension of the star-forming populations seen in the ACS field at lower surface brightness.

However, the lack of young stars does not preclude contamination from an extended disk component. Many disk galaxies show red color gradients in their outskirts, signifying an older stellar population extending beyond the star-forming disk. The color difference between the RGB populations in the WFC3 and ACS fields (Figure 6) again implies that these two populations are not identical, but these differences are consistent, in principle, with observed metallicity gradients in galaxy disks. In the Milky Way, for example, metallicity gradients in young populations such as Cepheids (Pedicelli et al. 2009; Luck \& Lambert 2011) or open clusters (Friel et al. 2002; Sestito et al. 2008) range from -0.05 to -0.09 dex $\mathrm{kpc}^{-1}$, with suggestions of shallower gradients at large radius (beyond $10 \mathrm{kpc}$; e.g., Yong et al. 2005; Carraro et al. 2007; Sestito et al. 2008) or when traced by older populations (Cheng et al. 2012). In M101 itself, gas-phase oxygen abundances in $\mathrm{H}$ II regions have radial gradients of $-0.03 \mathrm{dex} \mathrm{kpc}^{-1}$ (Kennicutt et al. 2003; Croxall et al. 2016). This gradient is very similar to the metallicity gradient we would infer by comparing the RGB populations in the ACS and WFC3 fields $\left(d[M / H] / d r=-0.035 \mathrm{dex} \mathrm{kpc}^{-1}\right)$, although the RGB metallicities themselves are down by $\sim 0.35$ dex as compared to the measured gas-phase metallicities.

Based on the stellar populations alone, therefore, it is hard to discriminate whether the WFC3 populations are indicative of a true halo population versus that of an extended outer disk. Morphologically, though, the deep surface photometry of $\mathrm{M}$ +13 shows that the outer disk is quite distorted, with no smooth outer component. While the disk shows a quasiexponential profile when azimuthally averaged, beyond about $14^{\prime}(28 \mathrm{kpc})$, there is little evidence for any azimuthal symmetry; the outer regions of the disk consist solely of the tidal plumes. Therefore, it is unlikely that the WFC3 field samples a dynamically relaxed outer disk that is easily characterized by a smooth metallicity gradient. If this field is sampling disk stars, it is more likely they would be stars scattered outwards by interactions. In principle, if the scattering time is longer than the ages of the helium-burning stars (i.e., greater than a few hundred Myr), only the RGB and AGB stars would be present in the CMD, such that the lack of young stars is not an ironclad argument against disk scattering. However, under that scenario, we would expect metallicities of the RGB stars in the field to reflect those found at smaller radius, like those in the ACS field or even more metal-rich stars from the inner disk. However, the color distributions of the RGB stars in the WFC3 and ACS fields are demonstrably different (as shown in Figures 6 and 7). Thus, while we cannot absolutely rule out a disk scattering origin for these stars, we find it more plausible that we are sampling stars tracing M101's halo population instead.

\subsection{AGB Stars in the WFC3 Field}

The CMDs shown in Figure 5 also clearly show the presence of AGB stars in both the ACS and WFC3 fields-more specifically, the thermally pulsing AGB (TP-AGB) stars that are more luminous than stars at the RGB tip. These are of particular interest in the WFC3 field, where the RGB stars alone provide little information on the timescale of star formation in the field. The presence of TP-AGB stars significant brighter than the RGB tip can be an indicator of an intermediate-age $(t \sim 1-10 \mathrm{Gyr})$ stellar population for metal-poor $([M / H]<-1)$ stars (e.g., Guarnieri et al. 1997; Gallart et al. 2005; Grebel 2007). Thus, their presence in the WFC3 field provides an age discriminant (albeit, an approximate one) for the old metal-poor population present in that field.

We first test the significance of this population through star counts in the WFC3 CMD. Above the RGB tip at $M_{\mathrm{F} 814 \mathrm{~W}}=-4.1$ lies a population of objects that extend to $M_{\mathrm{F} 814 \mathrm{~W}}=-5.5$, with colors ranging from that of the RGB tip to redder colors of $\mathrm{F} 606 \mathrm{~W}-\mathrm{F} 814 \mathrm{~W} \approx 2.0$. In this region of the CMD, we observe $N=22$ objects, but ignore the objects immediately around $M_{\mathrm{F} 814 \mathrm{~W}}=-4.1$ that are likely RGB stars. We can estimate the Milky Way foreground contamination using the TRILEGAL and Besançon models described in Section 3, and find $3.2 \pm 1.6$ (TRILEGAL) and $3.5 \pm 1.8$ (Besançon) objects, which compares favorably with the $N=4$ objects observed in the Abell 2744 background field. We thus find a background-corrected excess population of $N_{\mathrm{AGB}}=18 \pm 5$ objects (using Poisson uncertainties only) in the WFC3 CMD, or a $>3 \sigma$ detection of an AGB in this field.

While the small number of AGB stars precludes a detailed analysis, we can compare these objects to AGB populations seen in other resolved stellar populations to place rough constraints on the star formation history of the field. One simple metric is to measure the maximum brightness of the AGB stars above the TRGB $\left(\Delta_{814}\right)$, which should range brighter for younger stellar populations at fixed metallicity (e.g., Rejkuba et al. 2006). For example, in a detailed analysis of stellar populations in a dozen metal-poor galaxies taken from the ANGST sample (Dalcanton et al. 2009), Girardi et al. (2010) found AGB stars typically extending $\Delta_{814}=1.0-1.5 \mathrm{mag}$ above the TRGB. The galaxies in that sample have metallicities in the range $-1.7<[\mathrm{Fe} / \mathrm{H}]$ $<-1.1$, comparable to that we infer from the WFC3 RGB population, and show very little evidence for recent star formation, with $80-95 \%$ of the stars in the sample galaxies 
having formed more than $3 \mathrm{Gyr}$ ago. We measure a similar range of AGB luminosities in our WFC3 field, with $\Delta_{814}=1.0$ (or 1.4, if the two stars at $\mathrm{F} 814 \mathrm{~W}=23.7$ are included). Given the similar AGB extent in M101 compared to those in the Girardi et al. sample, the AGB stars seen in our M101 WFC3 field seem consistent with an intermediate stellar population age of $3 \mathrm{Gyr}$ or older, with no sign of more recent star formation.

The only difference we see between the M101 halo field and the ANGST galaxy subsample is the total number of AGB stars present. Girardi et al. (2010) find AGB-to-RGB fractions $\left(N_{\mathrm{AGB}} / N_{\mathrm{RGB}}\right)$ between 0.02 and 0.05 , where $N_{\mathrm{RGB}}$ is derived using counts within two magnitudes of the RGB tip. Measured in the same way, our WFC3 RGB contains $226 \pm 15$ objects, or a total (corrected for background) of $N_{\mathrm{RGB}}=188 \pm 17$. Given the $N_{\mathrm{AGB}}=18 \pm 5$ measured above, we derive $N_{\mathrm{AGB}} / N_{\mathrm{RGB}}=0.096 \pm 0.028$. This value is larger than that observed in the Girardi sample at the $2 \sigma$ level, and may hint at differences in stellar populations compared to those ANGST galaxies. However, given the small number of AGB stars seen in the WFC3 field, we are hesitant to attach too much significance to this result.

\subsection{The Properties of M101's halo}

If the WFC3 field is, in fact, tracing M101 halo stars, what inferences can be made about the nature of M101's halo in general? While the small FOV of the WFC3 field precludes us from giving a global view of the entire M101 halo, we attempt here to place our results in context with other spiral galaxy halos. The stellar halos of spiral galaxies (including the Milky Way and M31) have very diverse properties, with a wide range of mean metallicities, number density profiles, and metallicity gradients (e.g., Mouhcine et al. 2005a, 2005b; Monachesi et al. 2013, 2016; Ibata et al. 2014; Merritt et al. 2016; Harmsen et al. 2017) While there is no clear correlation of these properties with total galaxy mass or luminosity, there is a trend with the stellar halo mass, in the sense that lower-mass stellar halos have lower metallicities and weaker metallicity gradients (Harmsen et al. 2017). These trends show broad agreement with models in which the outer halos of galaxies are shaped by accretion. In such models, galaxies with more massive stellar halos (e.g., M31) have accreted more massive satellites, which increase the mean metallicity of the resulting halo population (e.g., Font et al. 2006; Cooper et al. 2010, 2013; Deason et al. 2016, D’Souza \& Bell 2018).

The low metallicity we infer for the WFC3 halo field is significantly more metal-poor than that typically observed in the outer halos of spiral galaxies to date. Adopting a typical halo value of $[\alpha / \mathrm{Fe}]=+0.3$, our metallicity estimate of $[M / H]=-1.7 \quad$ translates to $[\mathrm{Fe} / \mathrm{H}] \sim-1.9 \quad$ (Salaris et al. 1993; Streich et al. 2014), which is nearly 0.5-1 dex lower than most spiral galaxy halos; the Milky Way's halo is the only one known to approach these low metallicities (Harmsen et al. 2017). Some of the difference may be due to the different radial ranges probed; the Harmsen et al. sample is measured at $30 \mathrm{kpc}$, while our WFC3 field is further out at $47 \mathrm{kpc}$. Our analysis of the RGB colors in the WFC3 and ACS fields (Section 4.1 and Figure 7) suggests an upper limit of $\sim-0.02 \mathrm{dex} \mathrm{kpc}^{-1}$ for any metallicity gradient, which would still leave the M101 halo more metal-poor than most spiral galaxy halos studied to date. However, this low metallicity is consistent with expectations from the trend between halo metallicity and halo mass, given the estimates of a very low halo mass for M101 (van Dokkum et al. 2014; Merritt et al. 2016).

Low-mass stellar halos are also thought to have weaker metallicity gradients. Expressed in terms of an F606W - F814W color gradient in the RGB populations, galaxies in the Harmsen sample had color gradients of $\lesssim-0.004$ mag kpc$^{-1}$, with lowmass halos showing little or no color gradient. Our rough estimate of the maximum allowable RGB color gradient between the ACS and WFC3 fields suggests a gradient of $-0.007 \mathrm{mag} \mathrm{kpc}^{-1}$, larger than any of the gradients seen in the Harmsen et al. sample. Such a large gradient would seem indicative of a more massive stellar halo (such as that in M31), at odds with the low halo mass estimate for M101. However, a few considerations temper this result. First and foremost, the gradient we infer is an upper limit; the true gradient may be significantly smaller. Second, Elias et al. (2018) have used the Illustris simulations to argue that the nonspherical shapes of galaxy halos can effectively reduce the observed halo surface brightness profiles by as much as 2 mag $\operatorname{arcsec}^{-2}$ in face-on spirals; if so, the low M101 halo mass (inferred from surface photometry) may need to be revised upward and bring the inferred gradients into somewhat better agreement with the relationship between halo mass and color gradient noted by Harmsen et al. (2017).

In summary, the low metallicity inferred for the M101 halo from the RGB stars present in our WFC3 field is similar to that of the Milky Way's outer halo, and broadly consistent with expectations for galaxies with low halo mass and a quiet accretion history. For example, Elias et al. (2018) used the Illustris simulations to investigate the behavior of spirals with low stellar mass halos. In these simulations, the weak stellar halos of galaxies like the Milky Way and M101 form early, and they are dominated by the accretion of low-mass satellitesconsistent with the lower metallicity we see. Interestingly, these simulations also predict that the M101 halo will have had a stronger in situ halo component within $r \sim 45 \mathrm{kpc}$, which would have a higher metallicity and lead to a stronger metallicity gradient between the inner and outer halo. If so, this behavior may also explain the relatively large gradient we infer for the halo metallicities between the ACS and WFC3 fields.

\subsection{Comparing Constraints from Resolved Populations and Integrated Light}

Our imaging data also give us the opportunity to directly compare our results to those obtained from the broadband surface photometry of M101's outskirts described in M+13. Integrated light surface photometry has very different systematic uncertainties, compared to resolved stellar population studies-surface photometry contends with issues of sky subtraction, scattered light, and large-scale flat-fielding, while resolved population studies deal with uncertainties due to background contamination, field crowding, and often a limited field of view. Thus, comparing results from studies conducted in the same fields provides an important cross-check of systematic uncertainties between the two techniques.

The ACS field was chosen specifically to lie on the extended NE Plume identified in broadband imaging by $\mathrm{M}+13$. That study showed the plume to be blue, with a total integrated $B-V$ color of $0.21 \pm 0.05$; coupled with the plume's faint luminosity and extremely low rate of star formation (assessed by the far-UV flux), $\mathrm{M}+13$ inferred the stellar populations in the plume formed during 
a weak burst of star formation that peaked $\approx 250-350$ Myr ago and has since largely died out. The picture is well-supported by the Hubble data presented here: the presence of the co-evol lump population seen in the ACS CMD also implies a fading burst population of roughly similar age. However, the Hubble CMD sharpens the view significantly, revealing the old RGB population as well as tracing the main sequence stars and helium-burning populations. On balance, the CMD modeling favors a slightly older age ( $\sim 300-400 \mathrm{Myr})$ for the starburst, along with a more slowly decaying star formation rate than the Gaussian model adopted in $\mathrm{M}+13$, but the CMD model that best matches the population mix also matches the broadband color and surface brightness of the plume measured over the ACS field of view (Table 1). The Hubble data also break the age-metallicity degeneracy of broadband colors, revealing the metal-poor nature of both the young and old stellar populations in the field.

In contrast, the WFC3 pointing targeted a region where no broadband light was detected in the $\mathrm{M}+13$ imaging, putting an upper limit on the surface brightness of $\mu_{V}>29.0 \mathrm{mag} \mathrm{arcsec}^{-2}$. In this region, the Hubble CMD reveals an old stellar population, but with very low surface density. Adopting a single burst stellar population model to match the star counts measured along the RGB, we infer (after photometric transformations, see Section 4.2) an integrated surface brightness of $\mu_{V}=30.86 \mathrm{mag} \mathrm{arcsec}^{-2}$, nearly 2 mag $\operatorname{arcsec}^{-2}$ fainter than the upper limit measured from the broadband imaging of $\mathrm{M}+13$. Thus, the sparse halo population we detect in the WFC3 field is consistent with the non-detection of broadband light in the field by $\mathrm{M}+13$.

Similarly deep imaging of M101 was used by van Dokkum et al. (2014) and Merritt et al. (2016) to construct an azimuthally averaged surface brightness profiles of M101 extending beyond the disk and into the stellar halo. At the 23!3 distance of our WFC3 field, those profiles show a surface brightness of $\mu_{g} \approx 32-33$ mag $\operatorname{arcsec}^{-2}$. Converting our inferred F814W surface brightness to $g$ yields $\mu_{g}=31.1 \mathrm{mag} \mathrm{arcsec}^{-2}$, or 1-2 mag arcsec ${ }^{-2}$ brighter than the azimuthally average profile. The source of this discrepancy remains unclear. One possibility is substructure within M101's halo. Deep imaging of many galaxies has revealed a wealth of accretion shells and plumes in their stellar halos (e.g., Martínez-Delgado et al. 2008, 2010; Janowiecki et al. 2010; Ibata et al. 2014); if our WFC3 field has serendipitously fallen on such a feature, we would infer a higher surface brightness than the azimuthally averaged value. At such depths, where galaxy halos are thought to dominated by accretion, such substructure could be significant (Bullock \& Johnston 2005; Cooper et al. 2013; Gómez et al. 2013), making azimuthal averaging highly uncertain (see also the discussion in Watkins et al. 2016). Conversely, the disagreement may simply be a sign of the systematic uncertainties inherent to surface photometry at such faint light levels, where slight differences in sky estimation and subtraction can lead to significant changes in the extracted surface brightness profiles. With only one pointing in M101's halo, it remains difficult to discern between these possibilities.

\subsection{What Happened to M101?}

Taken as a whole, the stellar populations in M101's outer disk provide important constraints on M101's dynamical history. The galaxy's lopsided disk and distorted outer isophotes have long suggested a recent interaction with one of its neighboring companions. The high-velocity HI gas observed in both the NE Plume and the E Spur (van der Hulst \& Sancisi 1988; Walter et al. 2008; Mihos et al. 2012) add to a picture in which an encounter has driven a strong response in the galaxy's morphology, kinematics, and star-forming history. However, the identity of the offending companion remains uncertain. One possibility is the dwarf irregular galaxy NGC 5477 , projected only $22^{\prime}(44 \mathrm{kpc}$ ) away from the center of M101. While its proximity is favorable for driving a local response in the galaxy's outer disk, it is difficult to see how such a low-luminosity dwarf could drive the strong global response of M101's asymmetric disk. A second candidate is the larger and more luminous companion NGC 5474, located 44' (88 kpc) to the south of M101. NGC 5474's lopsided morphology suggests it too has suffered a recent interaction, and extremely diffuse $\mathrm{HI}$ is seen between it and the larger M101 (Huchtmeier \& Witzel 1979; Mihos et al. 2012). Nonetheless, its distance from M101 and the lack of obvious tidal features even at faint surface brightness $(M+13)$ make the direct connection to M101's dynamical history still somewhat circumstantial.

Now, however, the coeval burst population seen in the CMD of the outer disk ACS field adds a new piece to the puzzle: the timescale since interaction. If the lump stars formed as a response to a tidal encounter, their age places the time since collision at $\Delta t \approx 300-400$ Myr. In loose groups, the typical encounter speed involving galaxies is approximately that of a marginally bound orbit, i.e., $v_{e} \approx \sqrt{2} \times v_{c}$, where $v_{c}$ is the circular velocity of the dominant galaxy, $\approx 200 \mathrm{~km} \mathrm{~s}^{-1}$ for M101 (Bosma et al. 1981). Therefore, the interacting companion would have traveled a distance $v_{e} \times \Delta t \approx 100 \mathrm{kpc}$ away from M101 in the time since closest approach. In projection, the separation could be significantly smaller, of course, but the rough agreement between the expected separation and NGC 5474's projected distance from M101 ( $88 \mathrm{kpc})$ lends support to a interaction scenario involving these two galaxies. The timescale argument also works against scenarios involving the smaller NGC 5477-while the dwarf is projected close to the NE Plume today, if the encounter happened $300 \mathrm{Myr}$ ago, as traced by the burst population, then given M101's rotation speed the outer disk has gone through one-third of a rotation since the burst was triggered. The observed proximity of NGC 5477 to the NE Plume may thus be mere happenstance, with the dwarf promoting only a weak perturbation at best, on M101's already damaged outer disk. To test this scenario and fully describe the dynamical evolution of the interactions between M101 and its companion galaxies, detailed simulations are clearly warranted.

\section{Summary}

Using deep imaging from the ACS and WFC3 cameras on $H S T$, we have studied stellar populations in the outskirts of the nearby spiral M101. We observed two fields: the ACS field targeted M101's outer disk (cospatial with the NE Plume discovered by Mihos et al. 2013) at a distance of $36 \mathrm{kpc}$ from the galaxy's nucleus, while the parallel WFC3 field was in an offset field sampling the galaxy's outer stellar halo at a distance of $47 \mathrm{kpc}$. Our photometry extends down to a limiting magnitude of $\mathrm{F} 814 \mathrm{~W}=28.6 \quad(50 \%$ completeness limit), tracing RGB stars 3.5 mag below the RGB tip, and probing deep enough to detect upper main sequence stars with ages as old as $\sim 400 \mathrm{Myr}$.

In the outer disk field probed by the ACS imaging, we find evidence of multiple stellar populations indicative of a complex and extended star formation history. In particular, we trace a population of evolved red giant branch stars, young main 
sequence stars, and blue and red helium-burning stars. The BHeB sequence shows a concentration of stars at $M_{\mathrm{F} 814 \mathrm{~W}}=-2.4$ and $\mathrm{F} 606 \mathrm{~W}-\mathrm{F} 814 \mathrm{~W}=0.22$, indicative of a co-evol stellar population likely made in a recent weak burst of star formation in M101's outer disk. Isochrone matching indicates an age of $\approx 300-400 \mathrm{Myr}$ and metallicity of $[M / H]=-1.15 \pm 0.2$ for this post-starburst population, while the older RGB stars appear to have a slightly lower metallicity of $[M / H]=-1.3 \pm 0.2$. These inferences are supported by CMD modeling using the IACstar package, which also shows that the burst population accounts for $\approx 10 \%$ of the stellar mass in the outer disk, and that the star formation rates have declined by roughly half since the onset of the burst.

In the offset WFC3 halo field, the CMD shows an old RGB sequence with no evidence for young stellar populations. The RGB in this field is $\sim 0.1$ mag bluer in F606W - F814W than that observed in the ACS disk field, implying a lower metallicity of $[M / H]=-1.7 \pm 0.2$. We also see a scattering of luminous stars on the AGB, suggesting the presence of an intermediate-age population. Comparisons with AGB populations found in old, metal-poor stellar halos (Girardi et al. 2010) suggest this AGB population is at least several Gyr old. The low metallicity of the M101 halo stars is similar to that of the Milky Way's outer halo, and consistent with models where low mass stellar halos like M101's are formed by early accretion events involving low-mass satellites, with very little accretion of massive objects at late times.

A comparison of these results from resolved stellar population work with those derived from deep surface photometry provides an important test of systematics between the two techniques. The stellar populations we observed in the ACS field confirm the inferences of a recent burst of star formation in M101's outer disk from our previous surface photometry $(\mathrm{M}+13)$. They also sharpen the view by revealing the presence of old RGB and AGB stars, constraining the metallicities of both the young and old stellar populations, and refining the recent evolution of the star formation rate in the outer disk. The very low spatial density of halo stars detected in the WFC3 field is consistent with the upper limit on the broad band halo light in the field by $\mathrm{M}+13$, but $1-2 \mathrm{mag} \mathrm{arcsec}^{-2}$ brighter than azimuthally averaged profiles published by van Dokkum et al. (2014) and Merritt et al. (2016). This discrepancy could be a signature of substructure within M101's halo, or due to unresolved systematics in the deep surface photometry.

In summary, we are left with a picture of M101 where a recent interaction, likely with the companion galaxy NGC 5474 , has stirred up gas in the outer disk and triggered a weak burst of star formation $\sim 300-400 \mathrm{Myr}$ ago, which is now fading out. These types of encounters are common in group environments (Sinha \& Holley-Bockelmann 2012), and by triggering these kinds of weak outer disk starburst events, they may lead to the slow growth of extended disks over time. At the same time, though, the low metallicity and low mass of M101's stellar halo argues that the galaxy has not experienced a significant number of massive accretion events in its evolutionary history. Thus, in the loose group environment where the dynamical timescales are long and mergers less common, late-type spirals like M101 (and perhaps the Milky Way as well) may walk an evolutionary path where their properties are shaped more by weak encounters rather than the massive accretion events that drive evolution in denser groups and clusters.

The authors would like to thank Andrew Dolphin for advice for the DOLPHOT reductions, as well as Tom Brown and Miranda Link for their assistance with the planning of the observations for this project. This research made use of Astropy, a community-developed core Python package for astronomy (Astropy Collaboration et al. 2013). J.C.M., P.R.D., and J.J.F. were supported through funds provided by NASA through grant GO-13701 from the Space Telescope Science Institute, which is operated by the Association of Universities for Research in Astronomy, Incorporated, under NASA contract NAS5-26555.

Workload for this project: J.C.M. and P.R.D. led the observational design, P.R.D. led the data reduction and the analysis of the WFC3 halo field, J.C.M. led the analysis of the ACS disk field and the CMD modeling, and J.J.F. led the reduction and analysis of the Abell 2744 flanking field for background estimation. P.H. and A.E.W. contributed to the analysis and interpretation of the data. J.C.M. led the writing of this manuscript, with contributions from all authors.

Facilities: HST, CWRU:Schmidt.

Software: DOLPHOT, Astropy.

\section{ORCID iDs}

\section{J. Christopher Mihos (ib https://orcid.org/0000-0002- 7089-8616}

Patrick R. Durrell (1) https://orcid.org/0000-0001-9427-3373

John J. Feldmeier (iD https://orcid.org/0000-0003-2908-2620

Paul Harding (ib https://orcid.org/0000-0003-3442-6248

\section{References}

Anderson, J., \& Bedin, L. R. 2010, PASP, 122, 1035

Aparicio, A., \& Gallart, C. 2004, AJ, 128, 1465

Asplund, M., Grevesse, N., Sauval, A. J., \& Scott, P. 2009, ARA\&A, 47, 481

Astropy Collaboration, Robitaille, T. P., Tollerud, E. J., et al. 2013, A\&A, 558, A33

Bakos, J., Trujillo, I., \& Pohlen, M. 2008, ApJL, 683, L103

Bernard, E. J., Ferguson, A. M. N., Chapman, S. C., et al. 2015, MNRAS, 453, L113

Bird, S., Harris, W. E., Blakeslee, J. P., \& Flynn, C. 2010, A\&A, 524, A71 Bohlin, R. C. 2016, AJ, 152, 60

Bosma, A., Goss, W. M., \& Allen, R. J. 1981, A\&A, 93, 106

Bresolin, F. 2017, in Outskirts of Galaxies, Vol. 434, ed. Knapen, Lee, \& Gil de Paz (Berlin: Springer), 145

Bresolin, F., Ryan-Weber, E., Kennicutt, R. C., \& Goddard, Q. 2009, ApJ, 695,580

Bressan, A., Marigo, P., Girardi, L., et al. 2012, MNRAS, 427, 127

Brooks, R. S., Wilson, C. D., \& Harris, W. E. 2004, AJ, 128, 237

Bruzzese, S. M., Meurer, G. R., Lagos, C. D. P., et al. 2015, MNRAS, 447, 618 Bullock, J. S., \& Johnston, K. V. 2005, ApJ, 635, 931

Bush, S. J., Kennicutt, R. C., Ashby, M. L. N., et al. 2014, ApJ, 793, 65 Caldwell, N. 2006, ApJ, 651, 822

Carraro, G., Geisler, D., Villanova, S., Frinchaboy, P. M., \& Majewski, S. R. 2007, A\&A, 476, 217

Castellano, M., Amorín, R., Merlin, E., et al. 2016, A\&A, 590, A31

Cheng, J. Y., Rockosi, C. M., Morrison, H. L., et al. 2012, ApJ, 746, 149

Cooper, A. P., Cole, S., Frenk, C. S., et al. 2010, MNRAS, 406, 744

Cooper, A. P., D’Souza, R., Kauffmann, G., et al. 2013, MNRAS, 434, 3348 Crnojević, D., Sand, D. J., Spekkens, K., et al. 2016, ApJ, 823, 19

Croxall, K. V., Pogge, R. W., Berg, D. A., Skillman, E. D., \& Moustakas, J. 2016, ApJ, 830, 4

Dalcanton, J. J., Williams, B. F., Lang, D., et al. 2012, ApJS, 200, 18

Dalcanton, J. J., Williams, B. F., Seth, A. C., et al. 2009, ApJS, 183, 67

Davidge, T. J. 2006, PASP, 118, 1626

Davidge, T. J. 2010, ApJ, 718, 1428 
Deason, A. J., Mao, Y.-Y., \& Wechsler, R. H. 2016, ApJ, 821, 5 Debattista, V. P., Mayer, L., Carollo, C. M., et al. 2006, ApJ, 645, 209 Dehnen, W., \& Binney, J. J. 1998, MNRAS, 298, 387 Dolphin, A. E. 2000, PASP, 112, 1383

D’Souza, R., \& Bell, E. F. 2018, MNRAS, 474, 5300

Durrell, P. R., Sarajedini, A., \& Chandar, R. 2010, ApJ, 718, 1118

Durrell, P. R., Williams, B. F., Ciardullo, R., et al. 2007, ApJ, 656, 746

Elias, L. M., Sales, L. V., Creasey, P., et al. 2018, arXiv:1801.07273

Ellis, S. C., \& Bland-Hawthorn, J. 2007, MNRAS, 377, 815

Erwin, P., Pohlen, M., \& Beckman, J. E. 2008, AJ, 135, 20

Fantin, N. J., Côté, P., Hanes, D. A., et al. 2017, ApJ, 843, 53

Ferguson, H. C., Dickinson, M., \& Williams, R. 2000, ARA\&A, 38, 667

Ferguson, H. C., Tanvir, N. R., \& von Hippel, T. 1998, Natur, 391, 461

Font, A. S., Johnston, K. V., Bullock, J. S., \& Robertson, B. E. 2006, ApJ, 638,585

Friel, E. D., Janes, K. A., Tavarez, M., et al. 2002, AJ, 124, 2693

Gallart, C., Zoccali, M., \& Aparicio, A. 2005, ARA\&A, 43, 387

Geller, M. J., \& Huchra, J. P. 1983, ApJS, 52, 61

Gil de Paz, A., Madore, B. F., Boissier, S., et al. 2005, ApJL, 627, L29

Gilbert, K. M., Kalirai, J. S., Guhathakurta, P., et al. 2014, ApJ, 796, 76

Girardi, L. 2016, AN, 337, 871

Girardi, L., Groenewegen, M. A. T., Hatziminaoglou, E., \& da Costa, L. 2005, A\&A, 436, 895

Girardi, L., Williams, B. F., Gilbert, K. M., et al. 2010, ApJ, 724, 1030

Gómez, F. A., Helmi, A., Cooper, A. P., et al. 2013, MNRAS, 436, 3602

Grebel, E. K. 2007, in Why Galaxies Care About AGB Stars: Their Importance as Actors and Probes 378, 375

Guarnieri, M. D., Renzini, A., \& Ortolani, S. 1997, ApJL, 477, L21

Harmsen, B., Monachesi, A., Bell, E. F., et al. 2017, MNRAS, 466, 1491

Harris, J., \& Zaritsky, D. 2009, AJ, 138, 1243

Harris, W. E. 1996, AJ, 112, 1487

Harris, W. E. 2010, arXiv:1012.3224

Harris, W. E., Durrell, P. R., Pierce, M. J., \& Secker, J. 1998, Natur, 395, 45 Huchtmeier, W. K., \& Witzel, A. 1979, A\&A, 74, 138

Ibata, R. A., Lewis, G. F., McConnachie, A. W., et al. 2014, ApJ, 780, 128

Illingworth, G. D., Magee, D., Oesch, P. A., et al. 2013, ApJS, 209, 6

Jang, I. S., \& Lee, M. G. 2015, ApJ, 807, 133

Janowiecki, S., Mihos, J. C., Harding, P., et al. 2010, ApJ, 715, 972

Jordi, K., Grebel, E. K., \& Ammon, K. 2005, AN, 326, 657

Kennicutt, R. C., \& Evans, N. J. 2012, ARA\&A, 50, 531

Kennicutt, R. C., Jr., Bresolin, F., \& Garnett, D. R. 2003, ApJ, 591, 801

Kroupa, P. 2001, MNRAS, 322, 231

Lee, M. G., \& Jang, I. S. 2016, ApJ, 831, 108

Lemonias, J. J., Schiminovich, D., Thilker, D., et al. 2011, ApJ, 733, 74

Lotz, J. M., Koekemoer, A., Coe, D., et al. 2017, ApJ, 837, 97

Luck, R. E., Gieren, W. P., Andrievsky, S. M., et al. 2003, A\&A, 401, 939

Luck, R. E., \& Lambert, D. L. 2011, AJ, 142, 136

Majewski, S. R., Schiavon, R. P., Frinchaboy, P. M., et al. 2017, AJ, 154, 94

Marigo, P., Girardi, L., Bressan, A., et al. 2017, ApJ, 835, 77

Martin, C. L., \& Kennicutt, R. C., Jr. 2001, ApJ, 555, 301

Martínez-Delgado, D., Gabany, R. J., Crawford, K., et al. 2010, AJ, 140, 962

Martínez-Delgado, D., Peñarrubia, J., Gabany, R. J., et al. 2008, ApJ, 689, 184

Martínez-Delgado, D., Pohlen, M., Gabany, R. J., et al. 2009, ApJ, 692, 955

Massey, R., Schrabback, T., Cordes, O., et al. 2014, MNRAS, 439, 887

Matheson, T., Joyce, R. R., Allen, L. E., et al. 2012, ApJ, 754, 19

McQuinn, K. B. W., Skillman, E. D., Cannon, J. M., et al. 2010, ApJ, 721, 297

McQuinn, K. B. W., Skillman, E. D., Dalcanton, J. J., et al. 2011, ApJ, 740, 48

McQuinn, K. B. W., Skillman, E. D., Dalcanton, J. J., et al. 2012, ApJ, 759, 77

Medina, G. E., Muñoz, R. R., Vivas, A. K., et al. 2018, ApJ, 855, 43

Merritt, A., van Dokkum, P., Abraham, R., \& Zhang, J. 2016, ApJ, 830, 62
Mihos, J. C., Harding, P., Spengler, C. E., Rudick, C. S., \& Feldmeier, J. J. 2013, ApJ, 762, 82

Mihos, J. C., Keating, K. M., Holley-Bockelmann, K., Pisano, D. J., \& Kassim, N. E. 2012, ApJ, 761, 186

Monachesi, A., Bell, E. F., Radburn-Smith, D. J., et al. 2013, ApJ, 766, 106

Monachesi, A., Bell, E. F., Radburn-Smith, D. J., et al. 2016, MNRAS, 457, 1419

Mouhcine, M., Ferguson, H. C., Rich, R. M., Brown, T. M., \& Smith, T. E. 2005a, ApJ, 633, 821

Mouhcine, M., Rich, R. M., Ferguson, H. C., Brown, T. M., \& Smith, T. E. 2005b, ApJ, 633, 828

Okamoto, S., Arimoto, N., Ferguson, A. M. N., et al. 2015, ApJL, 809, L1

Pedicelli, S., Bono, G., Lemasle, B., et al. 2009, A\&A, 504, 81

Peters, S. P. C., van der Kruit, P. C., Knapen, J. H., et al. 2017, MNRAS, 470, 427

Pietrinferni, A., Cassisi, S., Salaris, M., \& Castelli, F. 2004, ApJ, 612, 168

Radburn-Smith, D. J., de Jong, R. S., Seth, A. C., et al. 2011, ApJS, 195, 18

Radburn-Smith, D. J., Roškar, R., Debattista, V. P., et al. 2012, ApJ, 753, 138

Rejkuba, M., da Costa, G. S., Jerjen, H., Zoccali, M., \& Binggeli, B. 2006, A\&A, 448, 983

Rejkuba, M., Greggio, L., Harris, W. E., Harris, G. L. H., \& Peng, E. W. 2005, ApJ, 631, 262

Robin, A. C., Reylé, C., Derrière, S., \& Picaud, S. 2003, A\&A, 409, 523

Roškar, R., Debattista, V. P., Quinn, T. R., Stinson, G. S., \& Wadsley, J. 2008a, ApJL, 684, L79

Roškar, R., Debattista, V. P., Stinson, G. S., et al. 2008b, ApJL, 675, L65

Ruiz-Lara, T., Few, C. G., Gibson, B. K., et al. 2016, A\&A, 586, A112

Salaris, M., Chieffi, A., \& Straniero, O. 1993, ApJ, 414, 580

Schlafly, E. F., \& Finkbeiner, D. P. 2011, ApJ, 737, 103

Schmidt, F., Rozo, E., Dodelson, S., Hui, L., \& Sheldon, E. 2009, ApJ, 702, 593

Sellwood, J. A., \& Binney, J. J. 2002, MNRAS, 336, 785

Sestito, P., Bragaglia, A., Randich, S., et al. 2008, A\&A, 488, 943

Sinha, M., \& Holley-Bockelmann, K. 2012, ApJ, 751, 17

Sirianni, M., Jee, M. J., Benítez, N., et al. 2005, PASP, 117, 1049

Somerville, R. S., Lee, K., Ferguson, H. C., et al. 2004, ApJL, 600, L171

Streich, D., de Jong, R. S., Bailin, J., et al. 2014, A\&A, 563, A5

Thilker, D. A., Bianchi, L., Meurer, G., et al. 2007, ApJS, 173, 538

Trenti, M., \& Stiavelli, M. 2008, ApJ, 676, 767

Tully, R. B. 1988, Nearby Galaxy Catalog (Cambridge: Cambridge Univ. Press)

van der Hulst, T., \& Sancisi, R. 1988, AJ, 95, 1354

van Dokkum, P. G., Abraham, R., \& Merritt, A. 2014, ApJL, 782, L24

Villalobos, Á., \& Helmi, A. 2008, MNRAS, 391, 1806

Vlajić, M., Bland-Hawthorn, J., \& Freeman, K. C. 2009, ApJ, 697, 361

Vlajić, M., Bland-Hawthorn, J., \& Freeman, K. C. 2011, ApJ, 732, 7

Walker, I. R., Mihos, J. C., \& Hernquist, L. 1996, ApJ, 460, 121

Walter, F., Brinks, E., de Blok, W. J. G., et al. 2008, AJ, 136, 2563

Watkins, A. E., Mihos, J. C., \& Harding, P. 2016, ApJ, 826, 59

Watkins, A. E., Mihos, J. C., \& Harding, P. 2017, ApJ, 851, 51

Watkins, A. E., Mihos, J. C., Harding, P., \& Feldmeier, J. J. 2014, ApJ, 791, 38

Werk, J. K., Putman, M. E., Meurer, G. R., et al. 2010, AJ, 139, 279

Williams, B. F., Ciardullo, R., Durrell, P. R., et al. 2007, ApJ, 656, 756

Williams, B. F., Dalcanton, J. J., Johnson, L. C., et al. 2011, ApJL, 734, L22

Williams, B. F., Dalcanton, J. J., Seth, A. C., et al. 2009, AJ, 137, 419

Windhorst, R. A., Cohen, S. H., Hathi, N. P., et al. 2011, ApJS, 193, 27

Worthey, G., España, A., MacArthur, L. A., \& Courteau, S. 2005, ApJ, 631,820

Yong, D., Carney, B. W., \& Teixera de Almeida, M. L. 2005, AJ, 130, 597

Younger, J. D., Cox, T. J., Seth, A. C., \& Hernquist, L. 2007, ApJ, 670, 269

Zheng, Z., Thilker, D. A., Heckman, T. M., et al. 2015, ApJ, 800, 120 